\title{
Three carbon-enhanced metal-poor dwarf stars from the SDSS
}

\section{Chemical abundances from $\mathrm{CO}^{5} \mathrm{BOLD}$ 3D hydrodynamical model atmospheres ${ }^{\star}$}

\author{
N. T. Behara ${ }^{1,2,5}$, P. Bonifacio ${ }^{1,2,3}$, H.-G. Ludwig ${ }^{1,2,7}$, L. Sbordone ${ }^{1,2,6}$, J. I. González Hernández ${ }^{1,2,4}$, and E. Caffau ${ }^{2}$ \\ 1 CIFIST Marie Curie Excellence Team \\ e-mail: natalie.behara@obspm.fr \\ 2 GEPI, Observatoire de Paris CNRS, Université Paris Diderot, Place Jules Janssen, 92190 Meudon, France \\ 3 Istituto Nazionale di Astrofisica - Osservatorio Astronomico di Trieste, via Tiepolo 11, 34143 Trieste, Italy \\ 4 Dpto. de Astrofísica y Ciencias de la Atmósfera, Facultad de Ciencias Físicas, Universidad Complutense de Madrid, 28040 Madrid, \\ Spain \\ 5 Institut d'Astronomie et d'Astrophysique, Université Libre de Bruxelles, 1050 Bruxelles, Belgium \\ ${ }^{6}$ Max-Planck Institut für Astrophysik, Karl-Schwarzchild-Str. 1, 85741 Garching, Germany \\ 7 Zentrum für Astronomie der Universität Heidelberg, Landessternwarte, Königstuhl 12, 69117 Heidelberg, Germany
}

Received 31 August 2009 / Accepted 15 December 2009

\section{ABSTRACT}

\begin{abstract}
Context. The origin of carbon-enhanced metal-poor stars enriched with both $s$ and $r$ elements is highly debated. Detailed abundances of these types of stars are crucial to understand the nature of their progenitors.

Aims. The aim of this investigation is to study in detail the abundances of SDSS J1349-0229, SDSS J0912+0216 and SDSS J1036+1212, three dwarf CEMP stars, selected from the Sloan Digital Sky Survey.

Methods. Using high resolution VLT/UVES spectra $(R \sim 30000)$ we determine abundances for $\mathrm{Li}, \mathrm{C}, \mathrm{N}, \mathrm{O}, \mathrm{Na}, \mathrm{Mg}, \mathrm{Al}, \mathrm{Ca}, \mathrm{Sc}$, $\mathrm{Ti}, \mathrm{Cr}, \mathrm{Mn}, \mathrm{Fe}, \mathrm{Co}, \mathrm{Ni}$ and 21 neutron-capture elements. We made use of $\mathrm{CO}^{5} \mathrm{BOLD}$ 3D hydrodynamical model atmospheres in the analysis of the carbon, nitrogen and oxygen abundances. NLTE corrections for CI and OI lines were computed using the Kiel code. Results. We classify SDSS J1349-0229 and SDSS J0912+0216 as CEMP-r+s stars. SDSS J1036+1212 belongs to the class CEMPno/s, with enhanced $\mathrm{Ba}$, but deficient $\mathrm{Sr}$, of which it is the third member discovered to date. Radial-velocity variations have been observed in SDSS J1349-0229, providing evidence that it is a member of a binary system.

Conclusions. The chemical composition of the three stars is generally compatible with mass transfer from an AGB companion. However, many details remain difficult to explain. Most notably of those are the abundance of Li at the level of the Spite plateau in SDSS J1036+1212 and the large over-abundance of the pure $r$-process element Eu in all three stars.
\end{abstract}

Key words. stars: individual: SDSS J1349-0229 - stars: individual: SDSS J0912+0216 - stars: individual: SDSS J1036+1212 stars: abundances - stars: AGB and post-AGB - stars: atmospheres

\section{Introduction}

Carbon-enhanced metal-poor (hereafter CEMP) stars owe their name to a considerable overabundance of carbon with respect to iron $([\mathrm{C} / \mathrm{Fe}]>+1.0)$, and represent a sizeable fraction of the very metal-poor stars $([\mathrm{Fe} / \mathrm{H}]<-2.0)$. The frequency of CEMP stars has been estimated to be of the order of $14 \%$ by Cohen et al. (2005) and of $21 \%$ by Lucatello et al. (2006). This frequency appears to increase with decreasing metallicity, reaching approximately $40 \%$ for stars with $[\mathrm{Fe} / \mathrm{H}]<-3.5$ (Beers \& Christlieb 2005). The carbon excess in CEMP stars may stem from carbon production by nucleosynthesis in an asymptotic giant branch (AGB) star followed by mass transfer to a surviving companion. An alternative mechanism is the formation of the star from a C-enriched interstellar medium.

The chemical composition of these stars shows a considerable variety. On the basis of the abundance pattern of neutron capture elements Beers \& Christlieb (2005) suggested to divide CEMP stars into four classes: CEMP-no, CEMP-s, CEMP-r/s,

* Based on observations obtained with the ESO Very Large Telescope at Paranal Observatory, Chile (programmes 078.D-0217 and 383.D-0927).
CEMP-r. To these classes Sivarani et al. (2006) proposed to add the class of CEMP-no/s to accommodate two stars discovered in the course of the "First Stars" programme (see Bonifacio et al. 2009a, and references therein for a description of the programme). The CEMP-no stars do not show any enhancement of neutron-capture elements. The prototype of this class is CS 22957-027 (Norris et al. 1997; Bonifacio et al. 1998). The class also includes the three most Fe-poor stars known: HE 0107-5240, HE 1327-2326 and HE 0557-4840 (Christlieb et al. 2002; Frebel et al. 2005; Norris et al. 2007).

The majority of CEMP stars with $[\mathrm{Fe} / \mathrm{H}]>-3$ are strongly enhanced in $s$-process elements (Aoki et al. 2007) and form the class CEMP-s. Radial-velocity studies by Lucatello et al. (2005) have shown that these may all be members of binary systems, providing strong evidence for the mass transfer scenario from a more massive companion. The large scatter of observed $s$ element abundances may arise from the different masses of the companion stars, which presents a useful constraint for AGB nucleosynthesis models.

Among the stars with strong enhancement of the $s$-process elements, a high percentage also show large enhancements of $r$ process elements. These stars belong to the class CEMP-r/s, the 
Table 1. Log of observations.

\begin{tabular}{|c|c|c|c|c|c|c|}
\hline Star & $\begin{array}{ll}\alpha & \delta \\
& \\
(\mathrm{J} 2000) & \\
\end{array}$ & $\begin{array}{c}\text { Date and time } \\
\text { yyyy-mm-dd UT }\end{array}$ & $\begin{array}{l}\text { Exposure } \\
\text { time } \\
(\mathrm{s})\end{array}$ & Setting & $\begin{array}{l}\text { Heliocentric } \\
\text { radial velocity } \\
\mathrm{km} \mathrm{s}^{-1}\end{array}$ & $\begin{array}{r}g \\
\mathrm{mag} \\
\end{array}$ \\
\hline \multirow{5}{*}{ SDSS J1349-0229 } & $134913.5-022942.8$ & $2007-01-31 \quad 07: 32: 54$ & 3800 & Dic1 390+580 & 92.3 & 16.63 \\
\hline & & 2009-04-15 02:24:25 & 3005 & Dic2 346+760 & 121.8 & 16.63 \\
\hline & & 2009-04-15 03:54:58 & 3005 & Dic2 346+760 & 121.7 & 16.63 \\
\hline & & 2009-04-15 04:52:57 & 3005 & Dic2 346+760 & 122.0 & 16.63 \\
\hline & & 2009-04-15 05:45:07 & 3005 & Dic2 346+760 & 122.4 & 16.63 \\
\hline SDSS J0912+0216 & $091243.7+021623.7$ & 2006-12-31 07:41:05 & 3005 & Dic1 390+580 & 138.0 & 15.67 \\
\hline SDSS J1036+1212 & $103649.9+121219.8$ & 2007-01-29 07:16:27 & 3005 & Dic1 390+580 & -47.9 & 16.59 \\
\hline
\end{tabular}

prototypes of which (CS 22948-27 and CS 29497-34) were first studied by Barbuy et al. (1997) and Hill et al. (2000). Many scenarios have been proposed to explain the observed abundances. Most involve two independant processes, one for the $r$-elements and one for the $s$-elements. None of the scenarios, however, is entirely convincing given the many different abundance patterns observed in r/s stars as well as the uncertainties regarding the astrophysical sites of the $r$-process. For example, Johnson \& Bolte (2004) find r-element ratios which are difficult to explain with combinations of the normal $r$ and $s$-processes.

Only one $r$-process enhanced CEMP star (CEMP-r) has been detected: CS 22892-052 (Sneden et al. 2003). This stars exhibits the lowest carbon enhancement of all CEMP stars: $[\mathrm{C} / \mathrm{Fe}]=+1.0$. The $r$-process pattern observed in the heavier $n-$ capture elements was found to be in excellent agreement with the scaled solar $r$-process abundances. Lighter elements with $40 \leq Z \leq 50$ however were not found to match, leading the authors to suggest the existence of two $r$-process sites.

Finally the class of CEMP-no/s was up to now defined by only two members, CS 29528-041 and CS 31080-095, which show an enhancement of Ba over iron of about 1 dex, but a sizeable underabundance of $\mathrm{Sr}$.

The diversity of $s$ and $r$-element patterns among CEMP stars strongly suggests that this population of stars has several astrophysical origins. Other mechanisms may be responsible for their nucleosynthetic history beyond those involving AGB stars. Since the $s$-process and $r$-process occur under different physical conditions, they are likely to arise in different astrophysical sites.

In this paper we present detailed abundance analyses of three dwarf CEMP stars: SDSS J1349-0229, SDSS J0912+0216 and SDSS J1036+1212. These stars are extremely metal-poor, with $[\mathrm{Fe} / \mathrm{H}]<-2.50$, and were selected from our ongoing survey of extremely metal-poor dwarf candidates from the Sloan Digital Sky Survey. The first two belong to the CEMP-r+s class, while the third is the third known example of a CEMP-no/s star, after the first two discovered by Sivarani et al. (2006), suggesting that the class is probably not so rare.

\section{Target selection and observations}

We selected extremely metal-poor (EMP) dwarf candidates from the Sloan Digital Sky Survey (SDSS; York et al. 2000; Adelman-McCarthy et al. 2007; Adelman-McCarthy et al. 2008) with an automatic analysis code (Bonifacio \& Caffau 2003) in a version which provides estimates of $[\mathrm{M} / \mathrm{H}]$ from low resolution spectra. These candidates were observed at high resolution with the UVES spectrograph (Dekker et al. 2000) at the ESO $8.2 \mathrm{~m}$ Kueyen-VLT telescope as part of our survey of stars at low metallicity.
We observed 17 candidates; among these were the objects SDSS J1349-0229, SDSS J0912+0216 and SDSS J1036+1212. The SDSS spectra showed strong $G$ bands in these stars, and the weakness of the $\mathrm{Ca}$ II $\mathrm{K}$ lines indicated very low metallicities. Figure 1 displays the prominent $\mathrm{CH} G$ band of all three stars. This band is hardly detectable in non-C-enhanced stars of this effective temperature and metallicity. We thus targeted the three objects as CEMP stars. An analysis of the remaining 14 candidates will be presented in a future paper (Bonifacio et al. 2010).

The slit was set to 1".4 and the detector was read with a $2 \times 2$ on-chip binning. The resulting resolution is $R \sim 30000$. Supplementary observations were obtained for SDSS J1349-0229 in the ESO period 83 to study the UV OH lines and the permitted O I triplet at $777 \mathrm{~nm}$. Details on the settings and exposure times are listed in Table 1 along with measurements of the radial velocities. The second epoch observation of SDSS J1349-0229 reveals a large radial-velocity variation of the order of $30 \mathrm{~km} \mathrm{~s}^{-1}$, indicating clearly that it is a member of a binary system. The signal-to-noise ratio varies from 35 at $392 \mathrm{~nm}$ up to 110 above $650 \mathrm{~nm}$.

\section{Atmospheric parameters}

The effective temperatures of the stars were determined using both the wings of $\mathrm{H} \alpha$ and the Fe I excitation equilibrium. The surface gravity was derived from the $\mathrm{Fe} \mathrm{I} / \mathrm{Fe}$ II ionisation equilibrium, while the microturbulence was determined from Fe I lines. The FITLINE code (François et al. 2003) was used to measure the equivalent widths of the Fe I and Fe II lines. ATLAS model atmospheres and SYNTHE (Kurucz 1993, 2005b) synthetic spectra in their Linux version (Sbordone et al. 2004; Sbordone 2005) have been employed in the analysis, using scaled solar abundances with the exception of the alpha-elements, which were enhanced by 0.4 dex. The opacity distribution functions of Castelli \& Kurucz (2003) with a microturbulence of $1 \mathrm{~km} \mathrm{~s}^{-1}$ have been used. The $\mathrm{H} \alpha$ profiles were computed using the ATLAS9 models and a modified version of the BALMER code ${ }^{1}$. The self-broadening of $\mathrm{H} \alpha$ was treated with the Barklem et al. (2000b,a) theory, and for the Stark broadening the computations of Stehlé \& Hutcheon (1999) were used. Adopted stellar parameters are listed in Table 2.

The carbon and nitrogen abundances are significantly enhanced in these stars. In order to investigate the impact of the carbon abundance on the atmospheric structure, models were computed for our stellar parameters using ATLAS12 (Kurucz 1996, 2005a; Castelli 2005) with a carbon enhancement of $+3.0 \mathrm{dex}$,

\footnotetext{
${ }^{1}$ Provided by Cayrel and C. Van't Veer, the original BALMER code of R.L. Kurucz is available at http://kurucz .harvard.edu/
} 


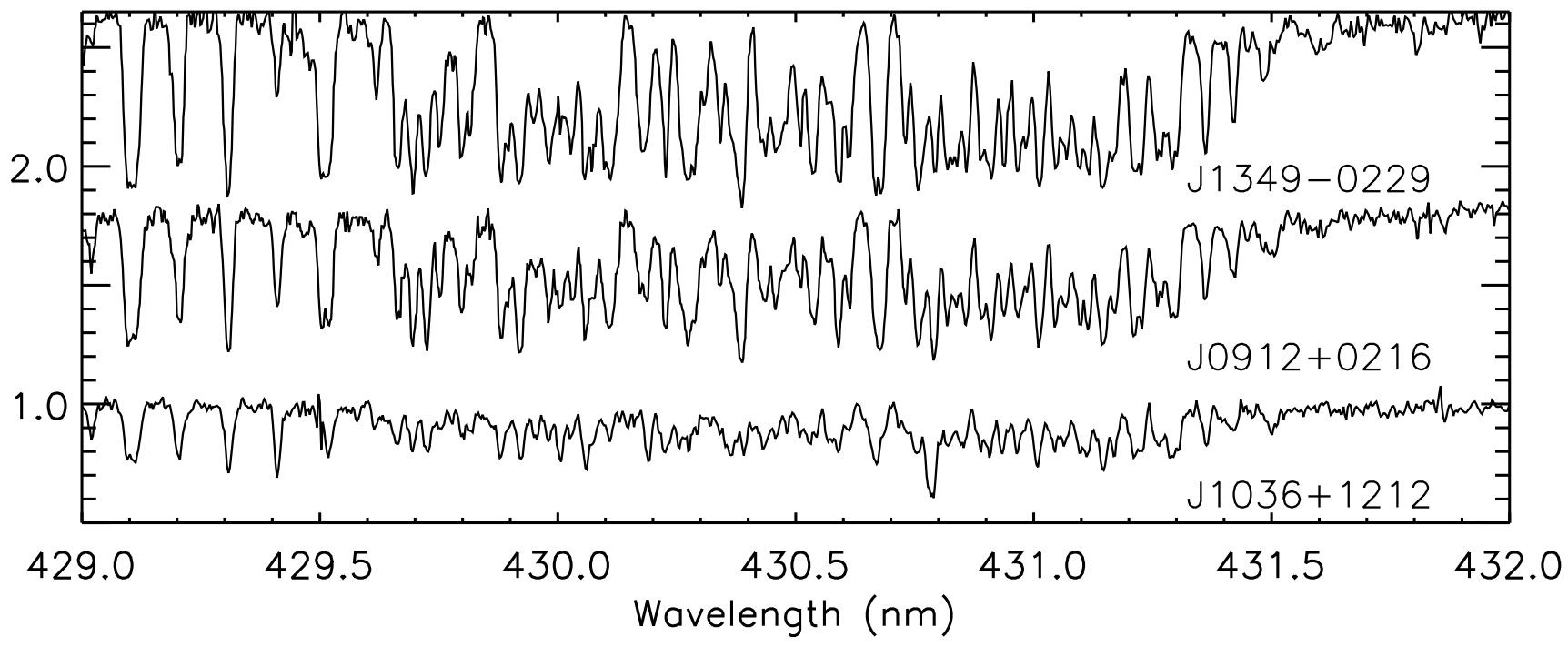

Fig. 1. Observed UVES spectra of the CH $G$ band in the three CEMP stars. Spectra have been offset for clarity.

and a nitrogen enhancement of $+2.0 \mathrm{dex}$. The average temperature difference in the two temperature structures is of the order of $20 \mathrm{~K}$. This small change in the temperature distribution translates into an error of approximately 0.02 dex in the abundance determination. The analysis was therefore performed using the ATLAS9 models.

\section{Abundances}

The majority of the elemental abundances presented were determined by the equivalent widths of unblended lines. A 1D LTE analysis was performed with the WIDTH9 code (Kurucz 1993, 2005a; Castelli 2005). The van der Waals damping constants have been taken from the Kurucz data base when available. When not available SYNTHE uses the WIDTH approximation (see Castelli 2005b; and also Ryan 1998). Abundances of strong lines, blended lines, or lines that are affected by hyperfine structure were treated by line-profile fitting, using the same code as in Caffau et al. (2005). The mean LTE 1D abundance for each of the 35 elements investigated in the three stars in our sample are presented in Table 3. In each line of the table we provide an estimate of the error for each star. For abundance indicators with multiple lines this is simply the standard deviation of the abundances derived from individual lines. For complex molecular bands like $\mathrm{C}_{2}$ the error has been estimated from the $\chi^{2}$ of the best fitting synthetic spectrum. The systematic errors for changes in effective temperature and surface gravity are similar to those of Sivarani et al. (2006): 0.10-0.15 dex in [X/Fe] for a change of $100 \mathrm{~K}$ in effective temperature or $0.5 \mathrm{dex}$ in $\log g$. As a reference we adopted the solar abundances derived with the use of the $\mathrm{CO}^{5}$ BOLD solar model, when available (Caffau et al. 2009), for other elements we adopted the values of Lodders (2003).

It is known that convection in metal-poor stars induces very low temperatures in the outermost layers, which are not predicted by classical 1D stellar atmospheres (Asplund et al. 1999; Collet et al. 2007; Caffau \& Ludwig 2007; González Hernández et al. 2008), this behaviour is often referred to as "overcooling". This is particularly important for molecular lines, which form high up in the atmosphere, where the overcooling is largest.
Table 2. Adopted stellar parameters.

\begin{tabular}{lcccc}
\hline \hline Star & $\begin{array}{c}T_{\text {eff }} \\
\mathrm{K}\end{array}$ & $\begin{array}{c}\log g \\
\mathrm{cgs}\end{array}$ & $\begin{array}{c}\xi \\
\mathrm{km} \mathrm{s}^{-1}\end{array}$ & $\begin{array}{c}{[\mathrm{Fe} / \mathrm{H}]} \\
\mathrm{dex}\end{array}$ \\
\hline SDSS J1349-0229 & 6200 & 4.0 & 1.5 & -3.0 \\
SDSS J0912+0216 & 6500 & 4.5 & 1.5 & -2.5 \\
SDSS J1036+1212 & 6000 & 4.0 & 1.4 & -3.2 \\
\hline
\end{tabular}

Investigations of $\mathrm{CH}, \mathrm{NH}$ and $\mathrm{OH}$ abundances in dwarf atmospheres with $[\mathrm{Fe} / \mathrm{H}]=-2.0$ by Asplund (2004) have shown that $1 \mathrm{D}$ models tend to overestimate abundances by more than 0.3 dex when compared to $3 \mathrm{D}$ hydrodynamical simulations. Collet et al. (2007) performed a similar investigation with red giant stars at $[\mathrm{Fe} / \mathrm{H}]=-3.0$ and found the corrections for $\mathrm{CH}$, $\mathrm{NH}$ and $\mathrm{OH}$ to be in the range of -0.5 to -1.0 dex.

It is thus mandatory to use 3D hydrodynamical simulations to assess molecular abundances in metal-poor stars. For this purpose, we employed 3D model atmospheres computed with the CO ${ }^{5}$ BOLD code (Freytag et al. 2002; Wedemeyer et al. 2004). The 3D spectral synthesis calculations were performed with the code Linfor3D ${ }^{2}$. The parameters of the 3D models used in the analysis are listed in Table 4 . The models were chosen from our 3D model grid (Ludwig et al. 2009) to best correspond with the stellar parameters of our three stars. Since we did not have a $3 \mathrm{D}$ model with $[\mathrm{Fe} / \mathrm{H}]=-2.5$, we interpolated for SDSS J0912+0216 between the corrections obtained from the models with $[\mathrm{Fe} / \mathrm{H}]=-2.0$ and $-3.0(\mathrm{~d} 3 \mathrm{t} 65 \mathrm{~g} 45 \mathrm{~mm} 20 \mathrm{n} 01$ and $\mathrm{d} 3 \mathrm{t} 65 \mathrm{~g} 45 \mathrm{~mm} 30 \mathrm{n} 01)$.

For each full 3D model, Linfor3D computes a temporal and horizontal average of the 3D structure over surfaces of equal (Rosseland) optical depth (hereafter denoted as $\langle 3 \mathrm{D}\rangle)$. We compared the abundances derived from each of our 3D models to this average model $\langle 3 \mathrm{D}\rangle$ and to a corresponding standard hydrostatic $1 \mathrm{D}$ model atmosphere $\left(1 \mathrm{D}_{\mathrm{LHD}}\right)$. The $1 \mathrm{D}_{\mathrm{LHD}}$ models are calculated assuming plane-parallel geometry and employ the

\footnotetext{
2 http://www .aip.de/ mst/Linfor3D/linfor_3D_manual. pdf
} 
A\&A 513, A72 (2010)

Table 3. Elemental abundances for the three CEMP stars obtained from an analysis using 1D stellar atmospheres.

\begin{tabular}{|c|c|c|c|c|c|c|c|c|c|c|}
\hline & \multicolumn{3}{|c|}{ SDSSJ1349-0229 } & \multicolumn{3}{|c|}{ SDSSJ0912+0216 } & \multicolumn{3}{|c|}{ SDSSJ1036+1212 } & \multirow{2}{*}{$\frac{\operatorname{Sun}}{\log (\epsilon)}$} \\
\hline Element & $\log (\epsilon)$ & {$[\mathrm{X} / \mathrm{Fe}]$} & $\sigma$ & $\log (\epsilon)$ & {$[\mathrm{X} / \mathrm{Fe}]$} & $\sigma$ & $\log (\epsilon)$ & {$[\mathrm{X} / \mathrm{Fe}]$} & $\bar{\sigma}$ & \\
\hline $\mathrm{Li}$ & & & & & & & 2.21 & & & \\
\hline $\mathrm{C}(\mathrm{CH})$ & 8.32 & 2.82 & 0.05 & 8.17 & 2.17 & 0.03 & 6.77 & 1.47 & 0.10 & 8.50 \\
\hline $\mathrm{C}(\mathrm{C} 2)$ & 8.66 & 3.16 & 0.02 & & & & & & & 8.50 \\
\hline C(C I $)$ NLTE & 7.92 & 2.42 & 0.11 & 7.38 & 1.38 & 0.05 & & & & 8.50 \\
\hline $\mathrm{N}(\mathrm{NH})$ & 6.46 & 1.60 & 0.12 & 7.11 & 1.75 & 0.12 & 5.95 & 1.29 & 0.25 & 7.86 \\
\hline $\mathrm{O}(\mathrm{OH})$ & 7.64 & 1.88 & 0.10 & & & & & & & 8.76 \\
\hline $\mathrm{O}(\mathrm{O}$ I) NLTE & 7.39 & 1.63 & 0.14 & & & & & & & 8.76 \\
\hline Na I NLTE & 4.79 & 1.49 & 0.01 & 4.18 & 0.38 & 0.03 & 3.53 & 0.43 & 0.01 & 6.30 \\
\hline Mg I & 5.12 & 0.57 & 0.16 & 5.26 & 0.21 & 0.13 & 4.35 & 0.00 & 0.06 & 7.55 \\
\hline Al I NLTE & 3.51 & 0.11 & $\ldots$ & 4.08 & 0.18 & $\ldots$ & 3.24 & 0.04 & $\ldots$ & 6.46 \\
\hline $\mathrm{Ca} \mathrm{I}$ & 3.74 & 0.40 & 0.08 & 3.32 & 0.42 & 0.12 & 3.52 & 0.38 & 0.17 & 6.34 \\
\hline Sc II & 0.08 & 0.01 & $\ldots$ & 0.85 & 0.28 & & -0.02 & 0.11 & 0.15 & 3.07 \\
\hline $\mathrm{Ti}$ I & & & & 2.96 & 0.54 & 0.03 & & & & 4.92 \\
\hline Ti II & 2.47 & 0.55 & 0.09 & 2.93 & 0.51 & 0.18 & 2.47 & 0.75 & 0.26 & 4.92 \\
\hline Cr I & 2.61 & -0.02 & 0.21 & 2.99 & -0.16 & 0.06 & 2.33 & -0.12 & 0.17 & 5.65 \\
\hline Cr II & 2.82 & 0.17 & . & 3.07 & -0.08 & 0.21 & 2.43 & -0.02 & 0.30 & 5.65 \\
\hline Mn I & 1.82 & -0.68 & 0.08 & 2.45 & -0.55 & $\ldots$ & & & & 5.50 \\
\hline Mn II & & & & 2.49 & -0.51 & 0.03 & 1.84 & -0.46 & 0.20 & 5.50 \\
\hline Co I & 2.24 & 0.33 & 0.19 & 2.72 & 0.31 & 0.15 & 2.28 & 0.57 & 0.09 & 4.91 \\
\hline Ni I & 3.48 & 0.26 & 0.37 & 3.79 & 0.07 & 0.18 & 3.30 & 0.28 & 0.22 & 6.22 \\
\hline Sr II & 1.21 & 1.30 & 0.17 & 0.98 & 0.57 & 0.07 & -0.85 & -0.56 & 0.04 & 2.91 \\
\hline Y II & 0.49 & 1.29 & 0.29 & 0.31 & 0.61 & 0.20 & -0.76 & 0.24 & 0.43 & 2.20 \\
\hline $\mathrm{Zr}$ II & 1.16 & 1.56 & 0.32 & 1.18 & 1.08 & 0.17 & 0.42 & 1.02 & 0.17 & 2.60 \\
\hline Ru I & & & & 1.93 & 2.61 & $\ldots$ & & & & 1.82 \\
\hline Pd I & & & & & & & 0.90 & 2.40 & 0.19 & 1.70 \\
\hline Ba II & 1.35 & 2.17 & 0.12 & 1.17 & 1.49 & 0.08 & 0.15 & 1.17 & 0.15 & 2.18 \\
\hline La II & -0.08 & 1.74 & 0.05 & 0.03 & 1.35 & 0.14 & 0.37 & 2.39 & 0.60 & 1.18 \\
\hline $\mathrm{Ce}$ II & 1.24 & 2.63 & 0.11 & 1.28 & 2.17 & 0.14 & 0.73 & 2.32 & 0.41 & 1.61 \\
\hline Pr II & 0.65 & 2.87 & 0.07 & 0.53 & 2.25 & 0.13 & 0.03 & 2.45 & 0.11 & 0.78 \\
\hline Nd II & 0.37 & 1.91 & 0.12 & 0.08 & 1.12 & 0.31 & 0.35 & 2.08 & 0.36 & 1.46 \\
\hline Sm II & 0.30 & 2.35 & 0.01 & 1.05 & 2.60 & 0.08 & 0.67 & 2.92 & 0.15 & 0.95 \\
\hline Eu II & -0.86 & 1.62 & 0.08 & -0.78 & 1.20 & 0.05 & -1.42 & 1.26 & 0.24 & 0.52 \\
\hline Gd II & 0.56 & 2.50 & 0.23 & 1.36 & 2.80 & 0.08 & 0.48 & 2.62 & 0.44 & 1.06 \\
\hline $\mathrm{Tb}$ II & 0.00 & 2.69 & 0.40 & 0.46 & 2.64 & 0.36 & 0.01 & 2.90 & 0.24 & 0.31 \\
\hline Dy II & 0.52 & 2.39 & 0.17 & 0.59 & 1.96 & 0.13 & 0.39 & 2.46 & 0.34 & 1.13 \\
\hline Er II & 0.68 & 2.73 & 0.08 & 0.48 & 2.03 & 0.23 & 0.61 & 2.86 & 0.04 & 0.95 \\
\hline Tm II & & & & & & & -0.31 & 2.78 & 0.13 & 0.11 \\
\hline Hf II & 1.01 & 3.14 & $\ldots$ & 1.09 & 2.72 & 0.19 & 0.02 & 2.35 & 0.60 & 0.87 \\
\hline Os II & & & & & & & 0.28 & 2.11 & $\ldots$ & 1.37 \\
\hline Ir I & & & & & & & 0.15 & 2.00 & $\ldots$ & 1.35 \\
\hline $\mathrm{Pb}$ & 2.14 & 3.09 & $\ldots$ & 1.88 & 2.33 & 0.30 & & & & 2.05 \\
\hline
\end{tabular}

same micro-physics (equation-of-state, opacities) as $\mathrm{CO}^{5} \mathrm{BOLD}$. Convection is described by a mixing-length theory in the formulation of Mihalas (1978); the adopted mixing-length-parameter was 0.5 .

Two main effects distinguish 3D models from 1D models: the average temperature profile and the horizontal temperature fluctuations. The contribution of both effects is quantified by the $3 \mathrm{D}$ correction in the sense 3D-1D. The correction attributed solely to the horizontal temperature fluctuations is denoted by $3 \mathrm{D}-\langle 3 \mathrm{D}\rangle$. A complete description of 3D corrections is given by Caffau et al. (2008) and references therein.

\subsection{Lithium}

The Li I $670.7 \mathrm{~nm}$ doublet was detected in SDSS J1036+1212, and an abundance of $A(\mathrm{Li})=2.21$ was derived. The doublet is not detected in SDSS J1349-0229 and SDSS J0912+0216.

The abundance of Li determined is quite high for this type of star, but "normal" for dwarf stars of this metallicity. In 2002, Reyniers et al. presented an alternative identification of the $\mathrm{Li}$ doublet in post-AGB stars: a Ce II line. Since SDSS J1036+1212
Table 4. Parameters of 3D models used in the analysis.

\begin{tabular}{lrlr}
\hline \hline Model & $T_{\text {eff }}$ & $\log g$ & {$[\mathrm{Fe} / \mathrm{H}]$} \\
\hline $\mathrm{d} 3 \mathrm{t} 63 \mathrm{~g} 40 \mathrm{~mm} 30 \mathrm{n} 01$ & 6270 & 4.00 & -3.0 \\
d3t65g45mm20n01 & 6530 & 4.50 & -2.0 \\
d3t65g45mm30n01 & 6550 & 4.50 & -3.0 \\
d3t59g40mm30n02 & 5850 & 4.00 & -3.0 \\
\hline
\end{tabular}

is strongly enhanced in neutron-capture elements (see Sect. 4.8), we investigated this possibility. The observed line is plotted in Fig. 2. Synthetic spectra were calculated with the abundance of Sm II and Ce II listed in Table 3 and three abundances of lithium: $\log \epsilon=1.00,2.00$ and 2.21.

Even with a significant enhancement, the contribution of the Sm II and Ce II lines are minimal. A stronger contribution from these lines would require a much lower surface gravity at this temperature. Additionally, we do not find that the line is shifted redwards compared to nearby lines, as was the case for the AGB stars of Reyniers et al. (2002). We can therefore confirm the identification of the observed feature with the $\mathrm{Li}$ I resonance 


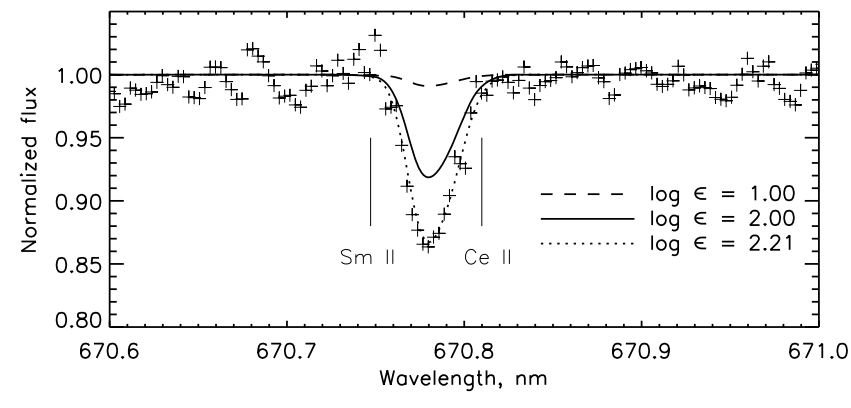

Fig. 2. Observed and synthetic spectra of SDSS J1036+1212 in the region of the Li I $670.7 \mathrm{~nm}$ doublet. Nearby lines of Ce II and Sm II were included in the synthesis. Synthetic spectra are shown with lithium abundances of $\epsilon=1.00,2.00$ and 2.21. See the text for details.

doublet and a lithium abundance of 2.21 for this star at our stellar parameters.

\subsection{Carbon}

Although in principle the carbon abundance of CEMP stars may be determined from both molecular and atomic lines, the majority of the values cited in the literature are derived from spectral lines of $\mathrm{CH}$ in the $G$ band, as these are strong features in CEMP star spectra. In some cases $\mathrm{C}_{2}$ features and $\mathrm{CI}$ lines may be present. We detect $\mathrm{CH}, \mathrm{C}_{2}$ and $\mathrm{CI}$ lines in SDSS J1349-0229 and $\mathrm{CH}$ and $\mathrm{CI}$ lines in SDSS J0912+0216. These stars exhibit a very strong carbon enhancement. In SDSS J1036+1212, the carbon enhancement is much weaker, and only $\mathrm{CH}$ lines could be used to determine $[\mathrm{C} / \mathrm{Fe}]$. The results are summarised in Table 5.

In order to perform $3 \mathrm{D}$ spectrum synthesis of molecular lines, which are computationally demanding, we decided to focus on four isolated $\mathrm{CH}$ features at $416.4 \mathrm{~nm}, 416.9 \mathrm{~nm}$, $418.0 \mathrm{~nm}$, and $418.8 \mathrm{~nm}$ to determine $[\mathrm{C} / \mathrm{Fe}]$ in all three stars. The line data were taken from the molecular line lists of Kurucz (Kurucz 2005b), but the oscillator strengths were scaled by a factor of 0.4 , as was done by Bonifacio et al. (1998) to place the $\log g f$ values on the same scale as the ones by Norris et al. (1997). From our 1D analysis we obtain $[\mathrm{C} / \mathrm{Fe}]=2.82 \pm 0.05,2.17 \pm 0.03$ and $1.47 \pm 0.10$ for SDSS J13490229, SDSS J0912+0216 and SDSS J1036+1212, respectively. The 3D corrections for these lines are quite substantial, have the effect to reduce the carbon abundance, and range from -0.73 to -0.50 dex.

A $1 \mathrm{D}$ carbon abundance of $[\mathrm{C} / \mathrm{Fe}]=3.16$ was derived from $\mathrm{C}_{2}$ features at $516.3 \mathrm{~nm}$ in the spectrum of SDSS J1349-0229. As for $\mathrm{CH}$, we used the molecular line lists of Kurucz (Kurucz $2005 \mathrm{~b}$ ) for the $\mathrm{C}_{2}$ lines. The $3 \mathrm{D}$ correction was computed from a synthesis of 118 lines centered at $516.3 \mathrm{~nm}$ and spanning $0.6 \mathrm{~nm}$. Observed and synthetic spectra of the feature are shown in Fig. 3. The 3D corrections for this feature are much more substantial than the corrections for the $\mathrm{CH}$ lines, they even reach -1.44 dex. This is because the $\mathrm{C}_{2}$ lines form in layers which are more superficial than those where the $\mathrm{CH}$ lines form, where the effects of overcooling and photospheric inhomogeneities are strongest.

Three CI lines were measured in SDSS J1349-0229 and two in SDSS J0912+0216. All of these lines are high excitation lines, and are highly sensitive to NLTE effects. Using LTE largely overestimates the abundance. The 3D corrections on the other hand, show that the 1D abundance is underestimated, however by a very small amount, the largest corrections being 0.08 dex. NLTE corrections were calculated using the Kiel

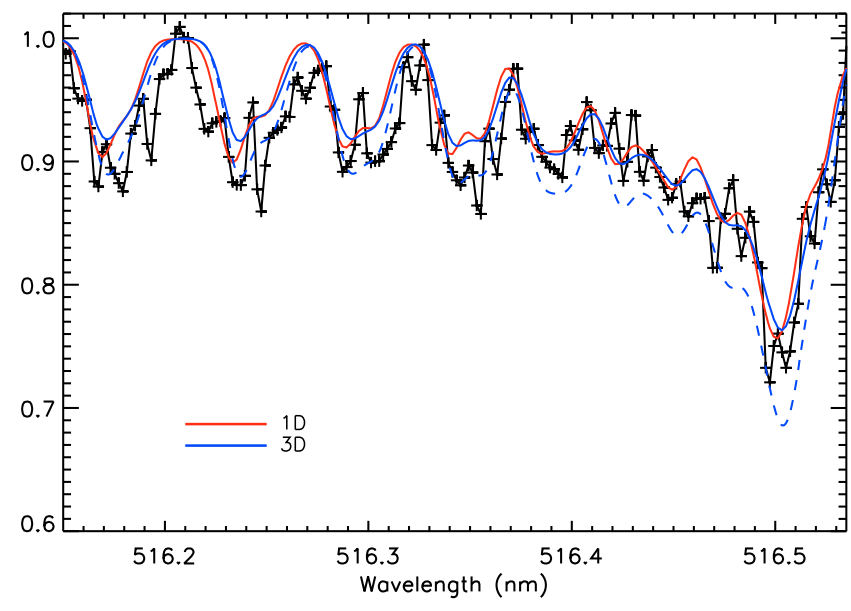

Fig. 3. Observed and 1D and 3D synthetic spectra of SDSS J1349-0229 in the region of the $C_{2}$ band at $516.3 \mathrm{~nm}$. The 3D spectra was computed with $[\mathrm{C} / \mathrm{Fe}]=1.72$ while the $1 \mathrm{D}$ spectra was computed with $[\mathrm{C} / \mathrm{Fe}]=3.16$. The dashed line shows the $3 \mathrm{D}$ synthetic spectra with an increase of 0.15 dex in carbon abundance.

code (Steenbock \& Holweger 1984) and the carbon model atom described in Stürenburg \& Holweger (1990), and are listed in Table 6. The effects of collisions with hydrogen atoms were treated as described in Steenbock \& Holweger (1984), who generalised the Drawin (1969) formalism. We provide NLTE corrections for three different values of $S_{\mathrm{H}}$, a scaling factor, $S_{\mathrm{H}}=0$ means totally neglecting collisions with $\mathrm{H}$ atoms, while $S_{\mathrm{H}}=1$ means taking the value of the Steenbock \& Holweger (1984) formalism. The NLTE CI abundance listed in Table 5 adopts the NLTE corrections for $S_{\mathrm{H}}=1 / 3$.

The three abundance indicators for SDSS J1349-0229 do not give consistent results either in the $1 \mathrm{D}$ or in the $3 \mathrm{D}$ analysis. The standard deviations of the abundances from the different features are 0.30 and 0.32 from the $1 \mathrm{D}$ and the 3D analyses, respectively. In 1D the most significant difference occurs between the $\mathrm{C}_{2}$ and $\mathrm{CI}$ lines, where we find a difference of 0.74 dex. In $3 \mathrm{D}$ the biggest difference of 0.79 dex occurs between the $\mathrm{CI}$ and $\mathrm{C}_{2}$ lines. The $\mathrm{CI}$ lines give a much higher abundance. Meanwhile the difference between 3D CH and CI is 0.42 dex, assuming an $S_{\mathrm{H}}=1 / 3$. For SDSS J0912+0216, where only $\mathrm{CH}$ and $\mathrm{CI}$ lines are available, we find differences of 0.79 and 0.23 dex and $\sigma=0.40$ and 0.12 in 1D and 3D, respectively.

The sensitivity of the molecular lines to temperature is clear from the significant 3D corrections. In Fig. 4 we have plotted the temperature distributions for the $3 \mathrm{D},\langle 3 \mathrm{D}\rangle$, and $1 \mathrm{D}_{\mathrm{LHD}}$ models. The ranges of the depth of formation, intended as the range in optical depth over which the line contribution function is significantly different from zero, of the $\mathrm{CH}, \mathrm{C}_{2}$ and $\mathrm{CI}$ lines are overplotted as horizontal lines. In $1 \mathrm{D}$ we derive a larger $\mathrm{C}$ abundance from the $\mathrm{C}_{2}$ lines than from the $\mathrm{CH}$ lines, while in $3 \mathrm{D}$ the reverse is true. This can be understood by looking at the different depths of formation of the $\mathrm{C}_{2}$ and $\mathrm{CH}$ lines in the $1 \mathrm{D}$ and $3 \mathrm{D}$ models. The $\mathrm{C}_{2}$ lines are formed higher up in the atmosphere compared to the $\mathrm{CH}$ lines, but far more in the 3D model than in the $1 \mathrm{D}$. The 3D models do not achieve a better consistency between $\mathrm{CH}$ and $\mathrm{C}_{2}$ lines. The $\mathrm{C}$ I lines are quite insensitive to $3 \mathrm{D}$ effects. Neither in 3D nor in 1D we achieved consistent results between molecular lines and CI.

In view of the high formation region of $\mathrm{C}_{2}$ we must consider the validity of the models in this region of the atmosphere. 


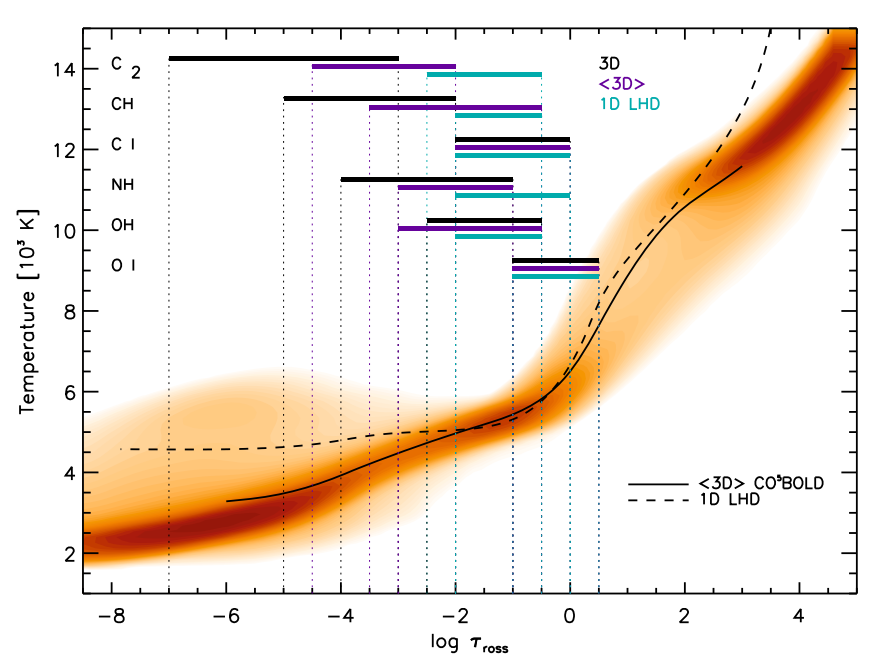

Fig. 4. The temperature structure for the 3D model d3t63g40mm30n01 is plotted as a function of $\tau_{\text {ross }}$ along with the average $3 \mathrm{D}(\langle 3 \mathrm{D}\rangle)$ and the corresponding $1 \mathrm{D}_{\mathrm{IHD}}$ temperature structures. Overplotted on the figure are the ranges of the depth of formation of the $\mathrm{C}_{2}, \mathrm{CH}, \mathrm{CI}, \mathrm{NH}, \mathrm{OH}$ and OI spectral lines used in the analysis.

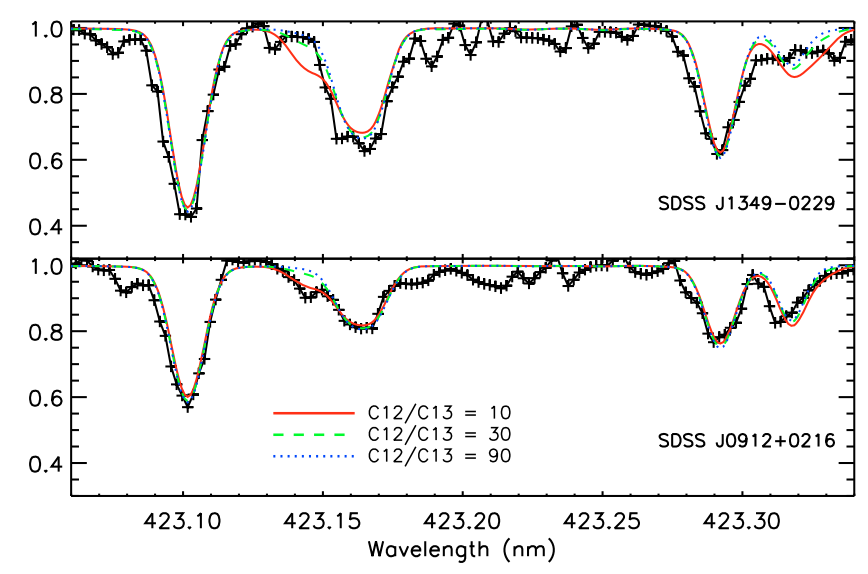

Fig. 5. Observed and synthetic spectra for two stars in the region of the ${ }^{13} \mathrm{CH}$ and ${ }^{12} \mathrm{CH}$ lines. Solid, dashed and dotted lines show the synthetic spectrum with a ratio of ${ }^{12} \mathrm{C} /{ }^{13} \mathrm{C}=10,30$ and 90 respectively.

The 3D model properties, like the choice of the opacity binning scheme, may well have an effect on the structure of the outer regions of the atmosphere. To explore this effect, we recomputed the carbon 3D corrections for SDSS J1349-0229 with a 3D model atmosphere that employs 12 opacity bins versus the six bins used in the models listed in Table 4. The 3D corrections are compared in Table 7 . The 12 bin model gives a better agreement between the $\mathrm{CH}$ and the $\mathrm{C}_{2}$ lines, and both molecular features agree better with $\mathrm{C}$ I. Although these results appear encouraging, the formation region of the $\mathrm{C}_{2}$ lines is quite high and at low density. The validity of LTE for molecule formation and levels population may well be an oversimplification. For further discussion on this topic see Behara et al. (2009).

\subsubsection{The ${ }^{12} \mathrm{C} /{ }^{13} \mathrm{C}$ ratio}

$\mathrm{CH}$ features from the $R$-branch of the $A^{2} \Delta-X^{2} \Pi$ band were used in our analysis of the ${ }^{12} \mathrm{C} /{ }^{13} \mathrm{C}$ isotopic ratio. Only lower limits could be derived: 30 for SDSS J1349-0229, and 10 for
SDSS J0912+0216. Observed spectra and fits to synthetic spectra are shown in Fig. 5. The low carbon abundance of SDSS J1036+1212 prevented us from obtaining a lower limit to the ${ }^{12} \mathrm{C} /{ }^{13} \mathrm{C}$ isotopic ratio.

\subsection{Nitrogen}

We obtain the nitrogen abundances for the three stars from isolated NH features at $\sim 336 \mathrm{~nm}$. We used the Kurucz data (Kurucz 2005b) for the NH molecule in our analysis, and following Spite et al. (2005) applied a correction of -0.40 dex to our abundances. The $1 \mathrm{D}$ analysis yields $[\mathrm{N} / \mathrm{Fe}]=1.60,1.75$ and 1.29 for SDSS J1349-0229, SDSS J0912+0216 and SDSS J1036+1212 respectively. The magnitude of the $3 \mathrm{D}$ corrections for $\mathrm{NH}$ is greater than the corrections for $\mathrm{CH}$ in all three stars and follows the same direction by reducing the $1 \mathrm{D}$ abundance. The corrections range from -0.67 to -0.93 . The adopted $3 \mathrm{D}$ abundances are listed in Table 5.

\subsection{Oxygen}

The oxygen forbidden lines at 630.0 and $636.4 \mathrm{~nm}$ are not detected in the spectra of our stars. Measured upper limits range from $[\mathrm{O} / \mathrm{Fe}]=2.0$ to 2.5 for the three stars. Spectra in the range of the UV OH lines and the permitted O I triplet at $777 \mathrm{~nm}$ were only available for SDSS J1349-0229, and therefore we can only determine the oxygen abundance for this star. We computed the abundance from three isolated UV OH lines of the (0-0) vibrational band of the $A^{2} \Sigma-X^{2} \Pi$ electronic systems, and computed the corresponding $3 \mathrm{D}$ corrections. The $g f$ values of the $\mathrm{OH}$ lines were computed from the lifetimes calculated by Goldman \& Gillis (1981). The result is $[\mathrm{O} / \mathrm{Fe}]=1.70$. We obtained a higher abundance from the triplet lines: $[\mathrm{O} / \mathrm{Fe}]=1.81$. However, it is well known that these high excitation lines suffer from NLTE effects (see e.g. Gratton et al. 1999 and references therein). We computed NLTE corrections using the Kiel code (Steenbock \& Holweger 1984) and the model-atom described in Paunzen et al. (1999). For oxygen the collisions with hydrogen atoms were treated with the Steenbock \& Holweger (1984) formalism for three choices of $S_{\mathrm{H}}$. The corrections are listed in Table 6. Taking this into account, we obtained an abundance from the triplet lines of 1.69 , which agrees excellently with the abundance obtained from the $\mathrm{OH}$ lines.

The 3D corrections for the $\mathrm{OH}$ lines are quite low $(\sim-0.2)$ compared to values found in the literature for metal-poor stars: Asplund \& García Pérez (2001) state values of less than -0.60 dex, and González Hernández et al. (2008) obtain corrections of -1.50 dex for dwarfs stars with $[\mathrm{Fe} / \mathrm{H}]=-3.00$. The $3 \mathrm{D}$ corrections for $\mathrm{OH}$ lines are very sensitive to the $\mathrm{C} / \mathrm{O}$ ratio in the atmosphere. We fixed $[\mathrm{C} / \mathrm{O}]=1.00$ in our calculations. Using a solar carbon-to-oxygen ratio yields a correction of -1.35 dex. Figure 6 shows the contribution function for a line computed for the two different $\mathrm{C} / \mathrm{O}$ ratios. When carbon is enhanced, there is no contribution to the $\mathrm{OH}$ line higher in the atmosphere, since the oxygen is tied up in $\mathrm{CO}$ in this region due to the high carbon content.

\subsection{The alpha elements $(Z>8)$}

Our measurements of $[\mathrm{Mg} / \mathrm{Fe}],[\mathrm{Ca} / \mathrm{Fe}]$ and $[\mathrm{Ti} / \mathrm{Fe}]$ in all three stars yield results that are consistent with the majority of metalpoor stars (Cayrel et al. 2004; Bonifacio et al. 2009b). 


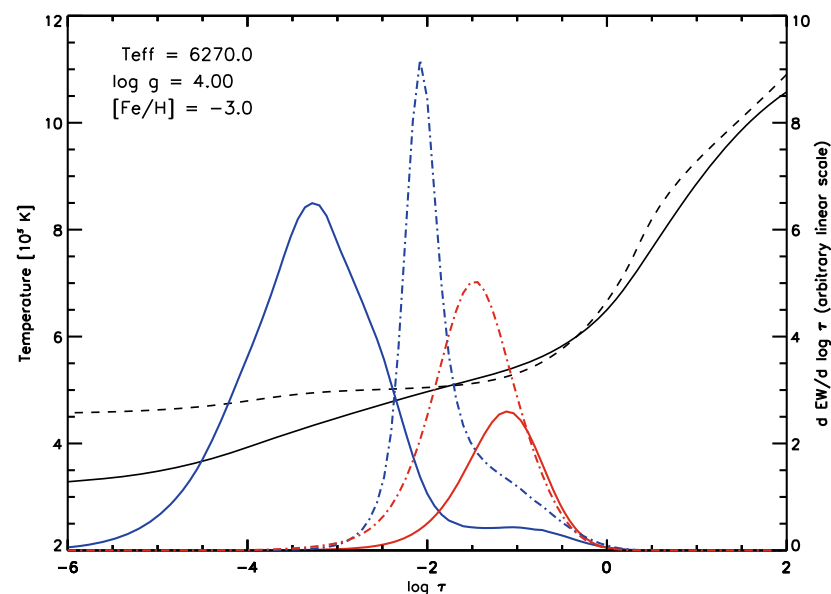

Fig. 6. Equivalent width contribution function plotted as a function of optical depth for the 3D model (blue) and the $1 \mathrm{D}_{\mathrm{LHD}}$ model (red) for two different $\mathrm{C} / \mathrm{O}$ ratios. Scaled solar carbon-to-oxygen is plotted as a solid line, while $[\mathrm{C} / \mathrm{O}]=1$ is plotted as a dot-dashed line. Overplotted are the temporal and horizontal average of the temperature profile of the 3D model (black solid line) and the temperature profile of the $1 \mathrm{D}_{\text {LHD }}$ model (dashed black line).

Table 5. Carbon, nitrogen and oxygen abundances and 3D corrections.

\begin{tabular}{lcccc}
\hline \hline Element & {$[\mathrm{X} / \mathrm{Fe}]_{1 \mathrm{D}}$} & {$[\mathrm{X} / \mathrm{Fe}]_{3 \mathrm{D}}$} & $3 \mathrm{D}-1 \mathrm{D}$ & $3 \mathrm{D}-\langle 3 \mathrm{D}\rangle$ \\
\hline & 2.82 & 2.09 & -0.73 & -0.37 \\
$\mathrm{CH}$ & 3.16 & 1.72 & -1.44 & -0.82 \\
$\mathrm{C}_{2}$ & 2.42 & 2.51 & 0.08 & -0.04 \\
$\mathrm{C}$ I NLTE & 1.60 & 0.67 & -0.93 & -0.34 \\
$\mathrm{NH}$ & 1.88 & 1.70 & -0.18 & -0.06 \\
$\mathrm{OH}$ & 1.63 & 1.69 & 0.06 & -0.04 \\
$\mathrm{O}$ I NLTE & \multicolumn{5}{c}{ SDSS J0912+0216 } \\
\hline CH & 2.17 & 1.67 & -0.50 & -0.20 \\
$\mathrm{C}$ I NLTE & 1.38 & 1.44 & 0.06 & -0.03 \\
$\mathrm{NH}$ & 1.75 & 1.07 & -0.67 & -0.30 \\
\hline \multirow{5}{*}{ SDSS J1036+1212 } \\
$\mathrm{CH}$ & 1.47 & 0.96 & -0.51 & -0.11 \\
$\mathrm{NH}$ & 1.29 & 0.51 & -0.78 & -0.08 \\
\hline
\end{tabular}

Notes. Abundances are expressed with respect to the solar abundances of $\log (\epsilon)=8.50,7.86$, and 8.76 for carbon, nitrogen and oxygen, respectively.

\subsection{The odd-Z elements}

Our measurement of $[\mathrm{Na} / \mathrm{Fe}]$ was made using the $\mathrm{Na} \mathrm{D}$ resonance lines at 588.995 and $589.592 \mathrm{~nm}$. We have applied NLTE corrections from Gratton et al. (1999). The corrections range from -0.07 to -0.15 dex. All stars show an enhancement of $\mathrm{Na}$, which is typical for CEMP stars (Stancliffe 2009).

The abundance of $\mathrm{Al}$ was measured from the $396.152 \mathrm{~nm}$ line. We found $[\mathrm{Al} / \mathrm{Fe}]$ to be nearly solar in all three stars after applying the NLTE correction from Baumüller \& Gehren (1997) of +0.64 , the mean value given for stars with $[\mathrm{Fe} / \mathrm{H}]<-2.20$ in their Table 2. This correction agrees closely with the NLTE correction given by Andrievsky et al. (2008) of 0.6 dex for stars with $[\mathrm{Fe} / \mathrm{H}]=-3.00$.

\subsection{The iron-peak elements}

The values of $[\mathrm{Sc} / \mathrm{Fe}],[\mathrm{Mn} / \mathrm{Fe}]$ and $[\mathrm{Co} / \mathrm{Fe}]$ all agree with typical values for metal-poor stars (Cayrel et al. 2004; Bonifacio et al. 2009b). The abundance of $\mathrm{Mn}$ and Co were calculated
Table 6. NLTE corrections for carbon and oxygen lines.

\begin{tabular}{lccc}
\hline \hline Line $(\mathrm{nm})$ & $S_{\mathrm{H}}=1.0$ & $S_{\mathrm{H}}=1 / 3$ & $S_{\mathrm{H}}=0.0$ \\
\hline \multicolumn{4}{c}{ SDSS J1349-0229 } \\
C I 493.2 & -0.352 & -0.432 & -0.502 \\
C I 505.2 & -0.369 & -0.456 & -0.535 \\
C I 538.0 & -0.379 & -0.468 & -0.543 \\
O I 777.1 & -0.127 & -0.144 & -0.130 \\
O I 777.4 & -0.098 & -0.111 & -0.099 \\
O I 777.5 & -0.100 & -0.113 & -0.101 \\
\hline \multicolumn{4}{c}{ SDSS J0912+0216 } \\
C I 505.2 & -0.267 & -0.352 & -0.456 \\
C I 658.8 & -0.258 & -0.335 & -0.425 \\
\hline
\end{tabular}

Table 7. 3D corrections calculated using 6 and 12 opacity bins.

\begin{tabular}{rccc}
\hline \hline 3D model & $\mathrm{CH}$ & $\mathrm{C}_{2}$ & $\mathrm{C}_{\text {I NLTE }}$ \\
\hline 6 bin $[\mathrm{X} / \mathrm{Fe}]_{3 \mathrm{D}}$ & 2.09 & 1.72 & 2.51 \\
12 bin $[\mathrm{X} / \mathrm{Fe}]_{3 \mathrm{D}}$ & 2.22 & 1.99 & 2.50 \\
\hline
\end{tabular}

using spectrum synthesis to take the effects of hyperfine splitting (HFS) into account. In the analysis we adopt the hfs line lists of Kurucz (2005b).

We were able to measure $\mathrm{Cr}$ I and $\mathrm{Cr}$ II lines in all stars. The abundances derived from the $\mathrm{Cr}$ II lines were found to be systematically higher than the values obtained from the $\mathrm{Cr}$ I lines. Bonifacio et al. (2009b) already noted this behaviour and suggested it may be due to NLTE effects. On average, we found a difference of 0.12 dex. The results of $\mathrm{Cr}$ I are consistent with the wide range of reported $\mathrm{Cr}$ abundances from CEMP stars though.

We note that Bergemann (2008) and Bergemann \& Gehren (2010) suggest that Mn, Co and Cr are affected by significant NLTE effects although no computations exist yet at a metallicity of -3 . In any case these corrections will be similar for CEMP stars and "normal" stars.

A wide range of $[\mathrm{Ni} / \mathrm{Fe}]$ values are found in CEMP stars. All our stars show an enhancement of $\mathrm{Ni}$, which ranges from +0.07 to +0.28 dex.

\subsection{Neutron-capture elements}

The spectra of all three stars display a wealth of neutron-capture element lines. All in all 21 elements are detected. $\mathrm{Pb}$ is detected in two stars, while in the third, SDSS J1036+1212, third peak $r$-process elements are detected.

We derive strontium abundances for our stars employing the strong Sr II $407.7 \mathrm{~nm}$ and $421.5 \mathrm{~nm}$ lines shown in Fig. 7. SDSS J1349-0229 and SDSS J0912+0216 exhibit overabundances of $\mathrm{Sr}$ of 1.30 and $0.57 \mathrm{dex}$, while SDSS J1036+1212 shows an underabundance of $\mathrm{Sr}$ by 0.56 dex. Zirconium and yttrium are found to be enriched in all stars.

Barium is overabundant in all three stars, although slightly less so in SDSS J1036+1212. The Ba abundances were estimated from the Ba II $493.4 \mathrm{~nm}$ and $614.1 \mathrm{~nm}$ lines. For the analysis we adopted the HFS by McWilliam (1998) and assumed a solar isotopic mix. We note however that if a pure $r$-process isotopic mix is assumed, the barium abundance is decreased by 0.1 to 0.35 dex.

Abundances from lanthanum lines were derived in all stars with the hyperfine structure constants and transition probabilities from Lawler et al. (2001a). The HFS components were calculated by the code LINESTRUC (Wahlgren 2005). 
A\&A 513, A72 (2010)
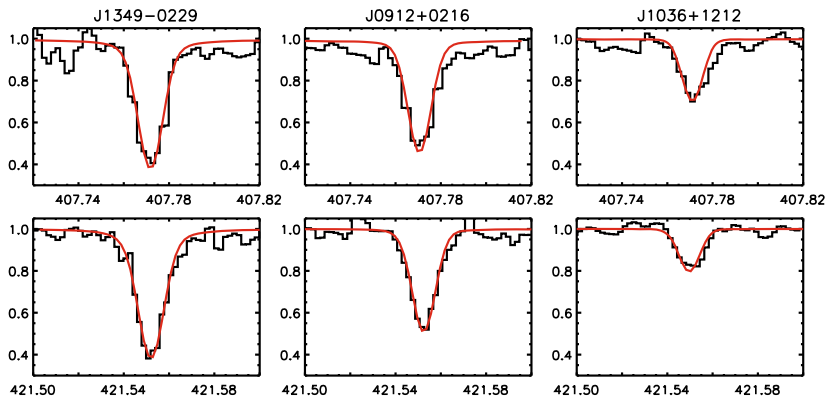

Fig. 7. Sr II $407.7 \mathrm{~nm}$ and $421.5 \mathrm{~nm}$ lines used in the abundance analysis.

For the cerium abundance analysis we adopted the line list from Lawler et al. (2009). The praseodymium abundance was derived using line lists from Li et al. (2007) and Ivarsson et al. (2001). Since the lines are relatively weak, we ignored any HFS. The linelist from Den Hartog et al. (2003) was used to determine the neodymium abundance.

For the analysis of the samarium lines we applied the values from Lawler et al. (2006). Europium lines were detected in all stars, and abundances were determined with the help of spectrum synthesis and line profile fitting, taking into account the HFS. Gadolinium line data were taken from Den Hartog et al. (2006), and data for terbium from Lawler et al. (2001b).

We ignored HFS in dypsrosium as the lines were weak. Oscillator strengths were adopted from Wickliffe et al. (2000). Data for erbium lines were taken from Lawler et al. (2008). For thulium we used the data from Wickliffe \& Lawler (1997). The effects due to HFS are small and were ignored. For the analysis of hafnium, we adopted values from Lawler et al. (2007).

Osmium and iridium lines were detected only in SDSS J1036+1212. Lead was detected in SDSS J1349-0229 and SDSS J0912+0216. The line list from Aoki et al. (2002) was used in the analysis.

\section{Comparison with similar stars}

It is now widely accepted that the CEMP class includes objects which have rather diverse astrophysical origin. In Sect. 1 we described the classification scheme proposed by Beers \& Christlieb (2005) based on the abundances of neutron-capture elements in CEMP stars. This classification is non-quantitative in the sense that one refers loosely to "enhancement" of $r$ or $s$-process elements without specifying which ones, and without providing quantitative limits for the enhancements. In the note to their Table 4, Sivarani et al. (2006) attempted to provide a more quantitative description of the classes, based essentially on the abundance of $\mathrm{Ba}$ and for one class (CEMP-s) also on the $\mathrm{Ba} / \mathrm{Eu}$ ratio.

Jonsell et al. (2006) defined the class CEMP-r+s, which is different from the class CEMP-r/s of Beers \& Christlieb (2005). In Table 8 we provide the definitions found in the literature of the different classes. All stars are assumed to have $[\mathrm{C} / \mathrm{Fe}]>+1$.

The classification scheme above is phenomenological and also leaves space for some ambiguity. For instance, all three of our programme stars belong to the CEMP-r+s class, but SDSS J1349-0229 also classifies as a CEMP-s (S2006) star. Moreover the really striking characteristic of the CEMP-no/s stars is that $[\mathrm{Ba} / \mathrm{Fe}]>0$ and $[\mathrm{Sr} / \mathrm{Fe}]<0$; if we redefine the class in this way, then SDSS J1036+1212 would classify as a CEMP-no/s star. Although our preferred carbon, nitrogen and oxygen abundances are those derived from the $3 \mathrm{D}$ analysis, we
Table 8. Classification of CEMP stars based on abundances of neutroncapture elements.

\begin{tabular}{lll}
\hline \hline Class & Definition & Reference \\
\hline CEMP-no & {$[\mathrm{Ba} / \mathrm{Fe}]<0.0$} & S2006 \\
CEMP-no/s & $+0.5<[\mathrm{Ba} / \mathrm{Fe}]<+1.0$ & S2006 \\
CEMP-s & {$[\mathrm{Ba} / \mathrm{Fe}]>+1.0$ and $[\mathrm{Ba} / \mathrm{Eu}]>+0.5$} & S2006 \\
CEMP-s & {$[\mathrm{Ba} / \mathrm{Fe}]>+1.0$ and $[\mathrm{Ba} / \mathrm{Eu}]>+0.0$} & $\mathrm{~J} 2006$ \\
& $\&[\mathrm{Eu} / \mathrm{Fe}]<+1.0$ & \\
CEMP-r $/ \mathrm{s}$ & $0.0<[\mathrm{Ba} / \mathrm{Fe}]<+0.5$ & S2006 \\
CEMP-r+s & {$[\mathrm{Ba} / \mathrm{Fe}]>1.0$ and $[\mathrm{Eu} / \mathrm{Fe}]>1.0$} & J2006 \\
\hline
\end{tabular}

Notes. S2006 and J2006 refer to Sivarani et al. (2006) and Jonsell et al. (2006), respectively.

adopt the abundances from the 1D analysis to compare our stars with a sample of hot dwarf CEMP stars from the literature. The full sample of stars selected is compiled from Table 10 of Aoki et al. (2008), Table 8 of Jonsell et al. (2006) and Table 4 of Sivarani et al. (2006).

\subsection{Lithium}

Few values of lithium have been measured in CEMP stars. Often only upper limits can be measured. Due to its fragility this element is an excellent diagnostic of the thermal history of the material. It is easily destroyed at temperatures above $2.5 \times 10^{6} \mathrm{~K}$, therefore material which has experienced these temperatures should be essentially Li-free. This could explain why CEMP stars generally show no $\mathrm{Li}$, as the temperatures necessary to produce the carbon will lead to Li destruction.

Still, a few CEMP stars have been observed to have Li values close to the value of the Spite plateau (Spite \& Spite 1982a,b). We present a sample of measured abundances in Fig. 8. A few stars show Li at the Spite plateau level, while others show a lower $\mathrm{Li}$ abundance. A remarkable example is the double lined spectroscopic binary CS 22964-161 (Thompson et al. 2008). The two stars are both CEMP, the primary is a warm sub-giant, while the secondary is a main sequence star. Li has been measured at the level of the Spite plateau in the primary star and is only tentatively detected in the secondary, again close to the Spite plateau. If binary mass transfer is responsible for the abundances of this system, it must once have been a triple system. That $\mathrm{Li}$ is detected at all in a star polluted by nuclearly processed material is in itself surprising, that it should be at the level of the Spite plateau is even more. There could of course be a conspiracy in a way that the Li produced in the donor star exactly matches the destroyed Li, modulo the dilution factor of the accreted material. In the case of CS 22964-161 one further requires that the dilution factor has been very nearly the same for both components of the binary. Lithium may be produced via several mechanisms during the AGB phase. However, if the observed lithium originates from the donor star, the question arises why Li abundances above the Spite plateau have not been detected.

We observe a $\mathrm{Li}$ abundance corresponding to the Spite plateau in SDSS J1036+1212. A scenario alternative to mass transfer in a binary star to explain the observed abundances would be the formation of SDSS J1036+1212 from a cloud of $\mathrm{C}$-rich material. Also in this case the $\mathrm{C}$-rich material should be essentially Li-free though, thus the Li-puzzle remains. Radial velocity monitoring of this star would be highly desirable to constrain the existence of a binary companion. 
N. T. Behara et al.: Spectral analyses of three CEMP stars

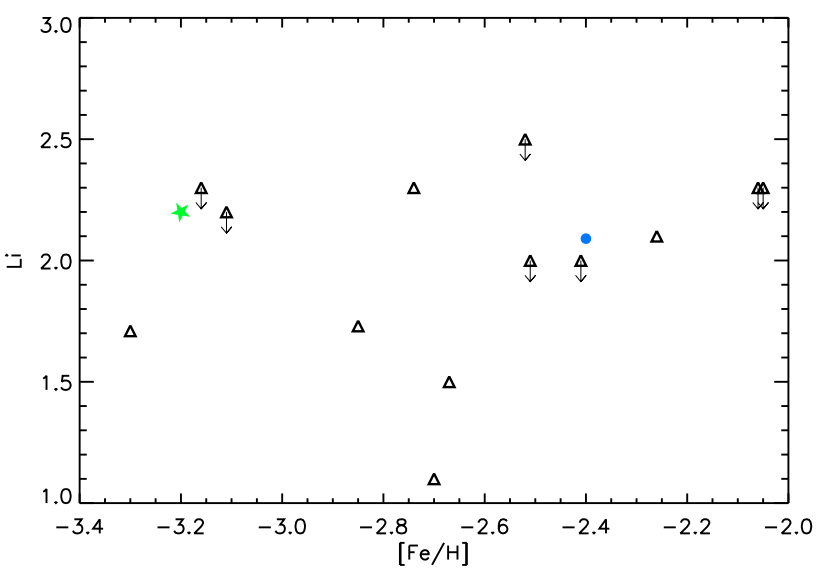

Fig. 8. Abundance of lithium as a function of $[\mathrm{Fe} / \mathrm{H}]$. Abundances from literature are plotted as open triangles, while the star symbol is from this work. The filled circle shows CS 22964-161.

\subsection{Carbon and nitrogen}

We plot $[\mathrm{C} / \mathrm{H}]$ as a function of $[\mathrm{Fe} / \mathrm{H}]$ in Fig. 9. The open symbols are from the literature, while the star symbols are from this work. Excluding three objects, SDSS J1036+1212 (this work), CS 29528-041 (Sivarani et al. 2006) and CS 22964-161 (Thompson et al. 2008), the stars display a rather constant $[\mathrm{C} / \mathrm{H}]$, slightly less than solar. A possible explanation is that such a roughly constant value is found when the accretion from the AGB star occurs through Roche lobe overflow. This suggests that the AGB companion always provides basically the same mass of carbon and the accretion mode through Roche lobe overflow ensures a roughly constant dilution factor. In this scenario stars with low carbon abundance, like SDSS J1036+1212, CS 29528041 and CS 22964-161 would arise through AGB wind instead from accretion, which implies larger dilution fractions.

There are three problems with this scenario: the first is that one would expect the plane $[\mathrm{C} / \mathrm{H}],[\mathrm{Fe} / \mathrm{H}]$ to be almost uniformly populated with different efficiencies due to a mixture of cases of Roche lobe overflow accretion and wind accretion, but the few "low" carbon stars cluster around $[\mathrm{C} / \mathrm{H}] \sim-1.5$. In the second place it is not at all clear that the carbon yield of AGB stars should be constant. Stars of different masses could provide different carbon abundances. From the theoretical point of view, while there are some discrepancies in the carbon yields for stars of a mass lower than $3 M_{\odot}$, there seems to be a general consensus that for higher mass AGB stars the carbon yield is roughly constant (Marigo 2001; Ventura et al. 2002; Herwig 2004; Karakas \& Lattanzio 2007). Thus our scenario is acceptable only if CEMP stars arise predominantly in binary systems in which the primary is more massive than $3 M_{\odot}$. It should be noted however that recently Ventura \& Marigo (2009) pointed out that AGB yields need to be computed including the changes in surface yields and their effect on opacities. Such a treatment may lead to a substantial revision of published yields. A third problem of our proposed scenario is that due to dilution; one should expect "low" C stars to show also lower abundances of n-capture elements, due to the larger dilution, but this is not so compelling for the three stars shown in Fig. 9. A larger sample of dwarf CEMP stars at $[\mathrm{Fe} / \mathrm{H}] \leq-3.2$ would be beneficial for a more complete discussion.

The nitrogen abundances are shown in Fig. 10 as a function of [C/Fe]. While CS 29528-041 exhibits a high $\mathrm{N}$ abundance for its low C abundance, SDSS J1036+1212 displays a nitrogen

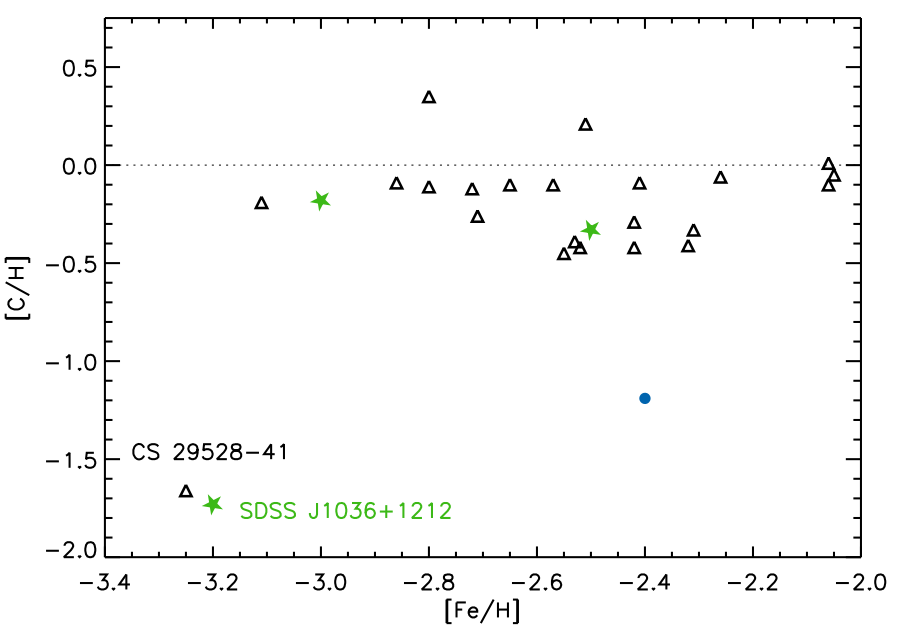

Fig. 9. Abundance of carbon as a function of $[\mathrm{Fe} / \mathrm{H}]$. Abundances from literature are plotted as open triangles, while star symbols are from this work. The filled circle shows CS 22964-161.

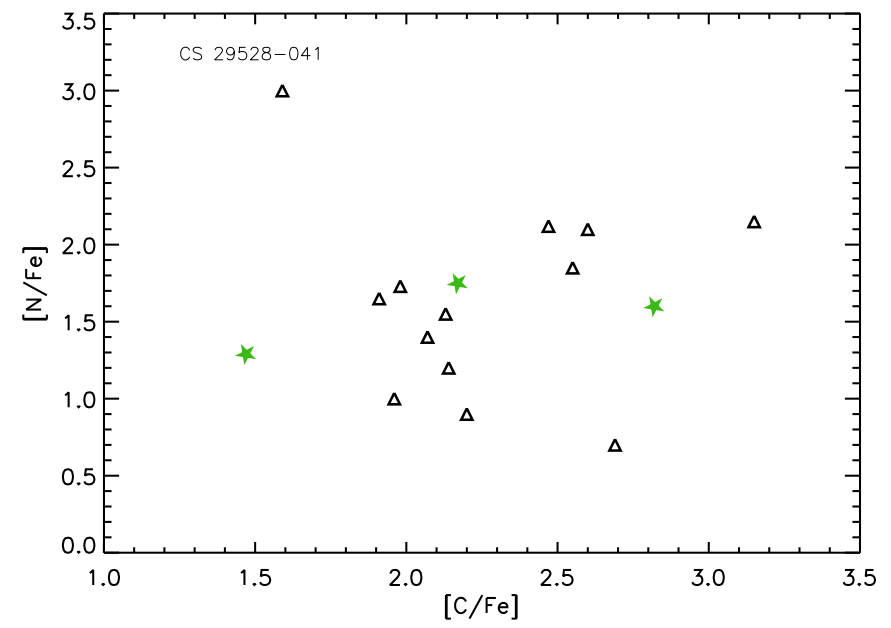

Fig. 10. Abundance of nitrogen as a function of $[\mathrm{C} / \mathrm{Fe}]$. Abundances from literature are plotted as open triangles, while star symbols are from this work.

abundance in the expected range, as do the other two stars in this work.

Our nitrogen abundances and lower limits on carbon isotopic ratios are compatible with the predictions of models of hot bottom burning in the envelopes of AGB stars (Karakas \& Lattanzio 2007). According to these authors intermediatemass low-metallicity AGB stars produce low $\mathrm{C} / \mathrm{N}$ and ${ }^{12} \mathrm{C} /{ }^{13} \mathrm{C}$ isotopic ratios, while low-mass $\mathrm{AGB}$ stars produce high $\mathrm{C} / \mathrm{N}$ and ${ }^{12} \mathrm{C} /{ }^{13} \mathrm{C}$ isotopic ratios. We measured $[\mathrm{C} / \mathrm{N}]=1.22$ for SDSS J1349-0229 and have a lower limit of 30 on its carbon isotopic ratio, qualitatively compatible with a low-mass AGB donor. We found a much lower value, $[\mathrm{C} / \mathrm{N}]=0.42$, for SDSS J0912+0216, and a lower limit on the carbon isotopic ratio of 10, compatible with an intermediate-mass AGB donor.

\subsection{Neutron-capture elements}

The abundance ratio of barium, representative of the $s$-process, to europium (representative of the $r$-process) is plotted in Fig. 11 as a function of metallicity. For comparison we have also 


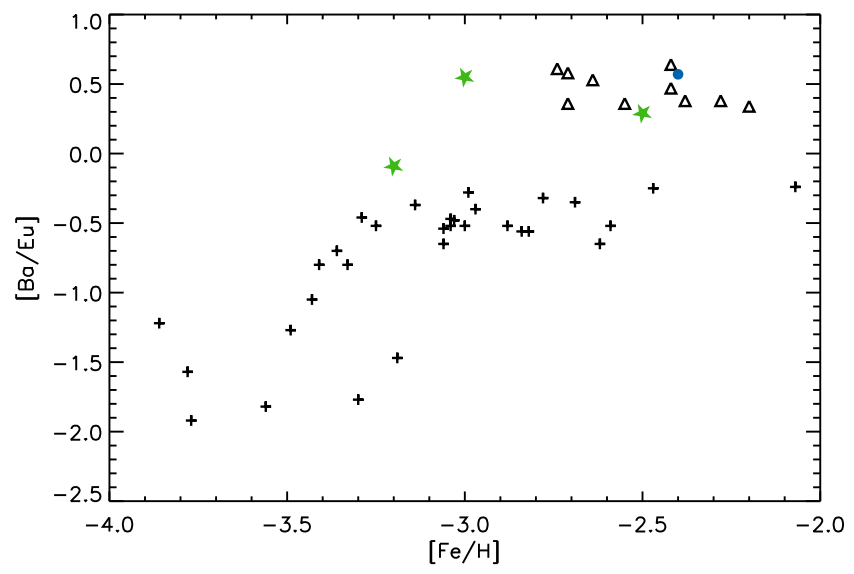

Fig. 11. $[\mathrm{Ba} / \mathrm{Fe}]$ as a function of $[\mathrm{Eu} / \mathrm{Fe}]$. Abundances from literature are plotted as open triangles, while star symbols are from this work. Included in this plot are EMP stars from François et al. (2007), plotted as crosses.

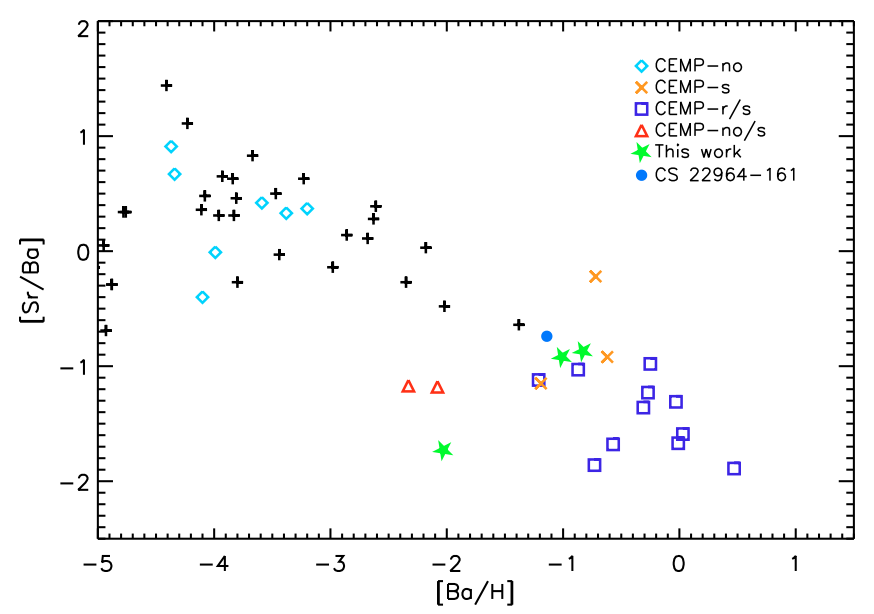

Fig. 12. The Sr/Ba ratio of our sample of stars compared to the classes of stars listed in Table 8 and the sample of extremely metal-poor stars from François et al. (2007).

plotted $[\mathrm{Ba} / \mathrm{Eu}]$ for the sample of extremely metal-poor stars from François et al. (2007). Here SDSS J1036+1212 separates itself again from the sample of CEMP-r/s stars, as the $[\mathrm{Ba} / \mathrm{Eu}]$ ratio is more similar to that observed in EMP stars, where we expect a pure $r$-process origin for the neutron-capture elements.

The $[\mathrm{Ba} / \mathrm{H}]$ can be used as an indicator of the $s$-process efficiency of the donor AGB star. We have plotted [ $\mathrm{Sr} / \mathrm{Ba}$ ] as a function of $[\mathrm{Ba} / \mathrm{H}]$ for the sample of stars in Fig. 12. A clear anti-correlation is seen between $[\mathrm{Sr} / \mathrm{Ba}]$ and $[\mathrm{Ba} / \mathrm{H}]$ as well as a progression from the stars with less $s$-process efficiency (CEMPno, EMP) to those with high efficiency (CEMP-r/s). The production ratio of heavy to light neutron-capture elements increases with total production efficiency.

We compared the element distributions of our three stars to theoretical model surface compositions of AGB stars. We employed models from Cristallo et al. (2009a,b). The results are shown in Figs. 13-15. The models were computed using the FRANEC code (Chieffi et al. 1998). The first model used in the comparison was computed with an initial mass of $2 M_{\odot}$ and $[\mathrm{Fe} / \mathrm{H}]=-2.14$. The second has an initial mass of $1.5 M_{\odot}$ and $[\mathrm{Fe} / \mathrm{H}]=-2.44$.

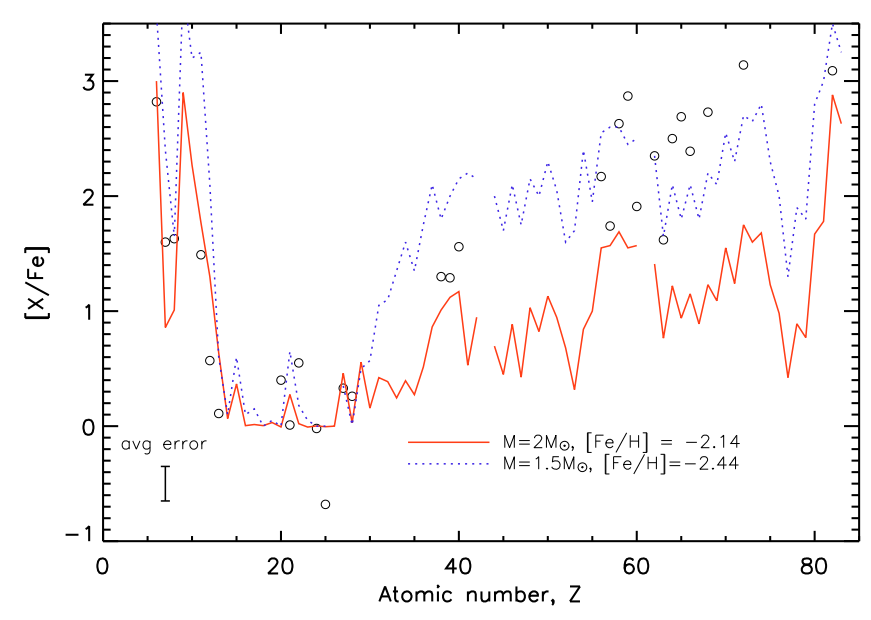

Fig. 13. Element distribution of SDSS J1349-0229 compared to model surface abundances of AGB stars. The first model, shown in red, has an initial mass of $2 M_{\odot}$ and $[\mathrm{Fe} / \mathrm{H}]=-2.14$ (Cristallo et al. 2009). The second model has an initial mass of $1.5 M_{\odot}$ and $[\mathrm{Fe} / \mathrm{H}]=-2.44$ (Cristallo et al. 2009b).

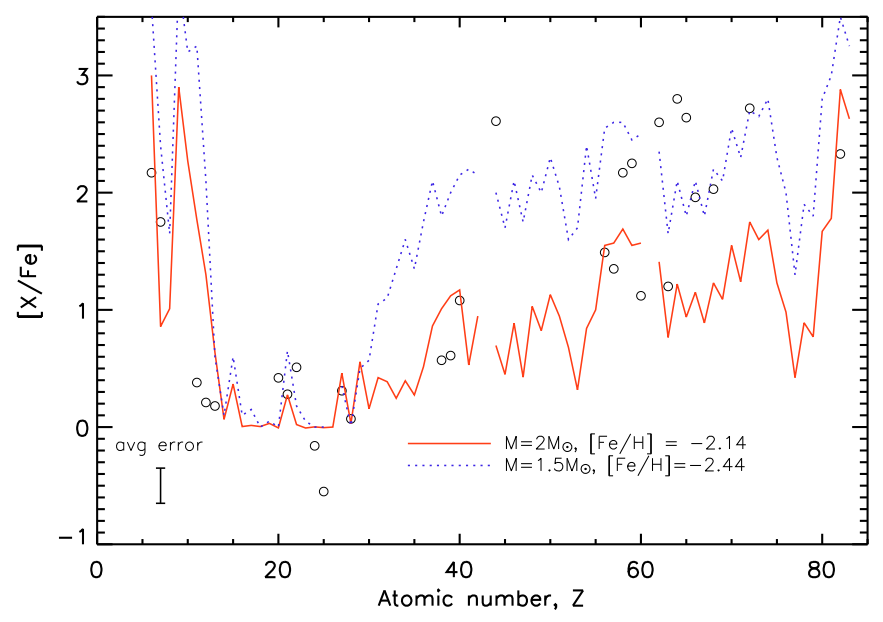

Fig. 14. As Fig. 13 for SDSS J0912+0216.

There is very little difference between the two models in the predicted distribution of the light elements, but larger $\mathrm{C}, \mathrm{N}$ and $\mathrm{O}$ values are produced in the model with the lower mass and metallicity. The models diverge at $Z>30$; the heavier elements are on average 1.0 dex more abundant in the lower metallicity model compared to the high metallicity, high mass model.

In general, the light $s$ elements ( $\mathrm{Sr}, \mathrm{Y}, \mathrm{Zr}$, hereafter $l s$ ) of the three stars are better reproduced by the more massive AGB model, while the heavy $s$ elements (Ba to Hf, hereafter $h s$ ) are better reproduced by the less massive AGB model. We are however limited by the two models used in our comparison. Bisterzo \& Gallino (2008) presented a comparison between a sample of 74 CEMP-s and CEMP-r/s stars and theoretical AGB models with different ${ }^{13} \mathrm{C}$-pocket efficiencies, initial masses $\left(M=1.3,1.5,2,3 M_{\odot}\right)$, and metallicities $(-3<[\mathrm{Fe} / \mathrm{H}]<-1)$. Their results showed that the low $[l s / \mathrm{Fe}]$ values found in CEMP stars can best be reproduced at low metallicities with lower initial AGB masses $\left(M<1.4 M_{\odot}\right)$. Additionally, a strong initial $r$ enrichment of the order of $[r / \mathrm{Fe}]=2.0 \mathrm{dex}$ was needed to match the observations of several CEMP-r/s stars. 


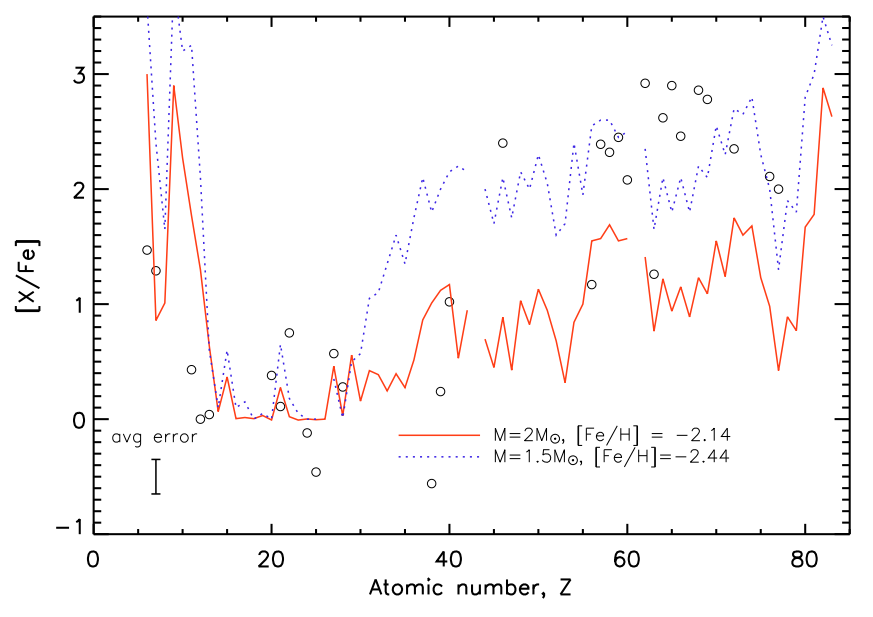

Fig. 15. As Fig. 13 for SDSS J1036+1212.

\section{Discussion and conclusions}

We classify SDSS J1349-0229 and SDSS J0912+0216 as CEMP-r+s stars. SDSS J1349-0229 shows clear radial-velocity variations following two sets of observations, which indicates that it is a member of a binary system. SDSS J1036+1212 shows similarities with CS 29528-041, a CEMP-no/s star. They have similar temperatures, gravities and metallicities. They also both stand out as carbon-poor with respect other CEMP stars. They differ in their nitrogen and $s$ and $r$-process abundances however. The $[h s / l s]$ ratio is higher for SDSS J1036+1212 compared to CS 29528-041, suggesting that the initial mass of the AGB companion star is lower for SDSS J1036+1212 than for CS 29528-041 (Bisterzo \& Gallino 2008). An AGB star with a higher initial mass would produce less heavy neutron-capture elements (see Figs. 13-15). We measured $[\mathrm{Ba} / \mathrm{Fe}]=1.17$ in SDSS J1036+1212, while the observed value in CS 29528-041 is 0.97. Furthermore, CS 29528-041 is extremely enhanced in nitrogen: $[\mathrm{N} / \mathrm{Fe}]=+3.07$, while SDSS J1036 +1212 is moderately enhanced with $[\mathrm{N} / \mathrm{Fe}]=+1.29$. High initial mass AGB models by Herwig (2004) with $M>4 M_{\odot}$ predict high nitrogen and low carbon, whereas the lower mass models predict lower nitrogen and high carbon. The high mass AGB models predict carbon abundances similar to those observed in these two stars. A plausible origin can therefore be determined for CS 29528-041 in pollution from a high mass AGB star. The elements observed in SDSS J1036+1212 point towards pollution by a low mass AGB companion. The low carbon abundance though is puzzling and cannot be explained by current models. Furthermore, the Li and $[\mathrm{Ba} / \mathrm{Eu}]$ is similar to that observed in EMP stars.

The scenario of mass transfer from an AGB companion is certainly the one which allows the explanation of most of the chemical abundances observed in the three stars studied in this work. The high $\mathrm{Li}$ abundance observed in one of them poses some problems: if it is attributed to a production in the AGB star, it requires some very fine tuning between $\mathrm{Li}$ production and destruction. Finally it is somewhat surprising that all three stars show a large (over 1 dex) enhancement of Eu, which is a pure $r$ process element. This does not fit the AGB nucleosynthesis and requires another origin. As all three stars show this signature, it implies that such an event is relatively common among CEMP stars.

Acknowledgements. We are grateful to R. Cayrel and C. Van't Veer for providing their modified version of the BALMER code and to P. François for providing us the FITLINE code. We acknowledge financial support from EU contract MEXT-CT-2004-014265 (CIFIST). We made use of the supercomputing centre CINECA, which has granted us time to compute part of the hydrodynamical models used in this investigation, through the INAF-CINECA agreement 2006, 2007. This research has made use of the SIMBAD database, operated at CDS, Strasbourg, France, and of NASA's Astrophysiscs Data System. Funding for the SDSS and SDSS-II has been provided by the Alfred P. Sloan Foundation, the Participating Institutions, the National Science Foundation, the U.S. Department of Energy, the National Aeronautics and Space Administration, the Japanese Monbukagakusho, the Max Planck Society, and the Higher Education Funding Council for England. The SDSS Web Site is http://www . sdss.org/. The SDSS is managed by the Astrophysical Research Consortium for the Participating Institutions. The Participating Institutions are the American Museum of Natural History, Astrophysical Institute Potsdam, University of Basel, University of Cambridge, Case Western Reserve University, University of Chicago, Drexel University, Fermilab, the Institute for Advanced Study, the Japan Participation Group, Johns Hopkins University, the Joint Institute for Nuclear Astrophysics, the Kavli Institute for Particle Astrophysics and Cosmology, the Korean Scientist Group, the Chinese Academy of Sciences (LAMOST), Los Alamos National Laboratory, the Max-Planck-Institute for Astronomy (MPIA), the Max-Planck-Institute for Astrophysics (MPA), New Mexico State University, Ohio State University, University of Pittsburgh, University of Portsmouth, Princeton University, the United States Naval Observatory, and the University of Washington.

\section{References}

Adelman-McCarthy, J. K., Jennifer, K., Agüeros, M. A., et al. 2007, ApJS, 172, 634

Adelman-McCarthy, J. K., Agüeros, M. A., Allam, S. S., et al. 2008, ApJS, 175, 297

Aoki, W., Ryan, S. G., Norris, J. E., et al. 2002, ApJ, 580, 1149

Aoki, W., Beers, T., Christlieb, N., et al. 2007, ApJ, 655, 492

Aoki, W., Beers, T., Sivarani, T., et al. 2008, ApJ, 679, 1351

Asplund, M. 2004, MmSAI, 75, 300

Asplund, M., \& Garcia Perez, A. E. 2001, A\&A, 372, 601

Asplund, M., Nordlund, A., Trampedach, R., \& Stein, R. 1999, A\&A, 346, L17

Barbuy, B., Cayrel, R., Spite, M., et al. 1997, A\&A, 317, L63

Barklem, P. S., Piskunov, N., \& O’Mara, B. J. 2000a, A\&A, 363, 1091

Barklem, P. S., Piskunov, N., \& O'Mara, B. J. 2000b, A\&A, 355, L5

Baumueller, D., \& Gehren, T. 1997, A\&A, 325, 1088

Beers, T. C., \& Christlieb, N. 2005, ARA\&A, 43, 531

Behara, N. T., Ludwig, H.-G., Bonifacio, P., et al. 2009, MmSAI, 80

Bergemann, M. 2008, Phys. Scr. T, 133, 014013

Bergemann, M., \& Gehren, T. 2010, in Chemical Abundances in the Universe:

Connecting First Stars to Planets, ed. K. Cunha, M. Spite, \& , B. Barbuy, IAU

Symp., 265, 348

Bisterzo, S., \& Gallino, R. 2008, AIPC, 1001, 131

Bonifacio, P., \& Caffau, E. 2003, A\&A, 399, 1183

Bonifacio, P., Molaro, P., Beers, T. C., \& Vladilo, G. 1998, A\&A, 332, 672

Bonifacio, P., Andersen, J., Andrievsky, S. M., et al. 2009a, in Science with VLT in the ELT era, ed. A. Moorwood (Berlin: Springer Verlag), 31

Bonifacio, P., Spite, M., Cayrel, R., et al. 2009b, A\&A, 501, 519

Caffau, E., \& Ludwig, H.-G. 2007, A\&A, 467, L11

Caffau, E., Bonifacio, P., Faraggiana, R., et al. 2005, A\&A, 441, 533

Caffau, E., Sbordone, L., Ludwig, H.-G., et al. 2008, A\&A, 483, 591

Caffau, E., Ludwig, H.-G., \& Steffen, M. 2009, MmSAI, 80

Castelli, F. 2005a, MSAIS, 8, 25

Castelli, F. 2005b, MSAIS, 8, 44

Castelli, F., \& Kurucz, R. L. 2003, in Modelling of Stellar Atmospheres, ed. N.

Piskunov, et al., IAU Symp., 210, Poster A20, [arXiv: astro-ph/0405087] Cayrel, R., Depagne, E., Spite, M., et al. 2004, A\&A, 416, 1117

Chieffi, A., Limongi, M., \& Straniero, O. 1998, ApJ, 502, 737

Christlieb, N., Bessell, M. S., Beers, T. C., et al. 2002, Nature, 419, 904

Cohen, J. G., Shectman, S., Thompson, I., et al. 2005, ApJ, 633, L109

Collet, R., Asplund, M., \& Trampedach, R. 2007, A\&A, 469, 687

Cristallo, S., Straniero, O., Gallino, R., et al. 2009a, ApJ, 696, 797

Cristallo, S., Piersanti, L., Straniero, O., et al. 2009b, PASA, 26, 139

Dekker, H., D’Odorico, S., Kaufer, A., Delabre, B., \& Kotzlowski, H. 2000, Proc. SPIE, 4008, 534

Den Hartog, E. A., Lawler, J. E., Sneden, C., \& Cowan, J. J. 2003, ApJS, 148, 543

Den Hartog, E. A., Lawler, J. E., Sneden, C., Cowan, J. J. 2006, ApJS, 167, 292

Drawin, H. W. 1969, Z. Phys., 225, 483

François, P., Depagne, E., Hill, V., et al. 2003, A\&A, 403, 1105

François, P., Depagne, E., Hill, V., et al. 2007, A\&A, 476, 935

Frebel, A., Aoki, W., Christlieb, N., et al. 2005, Nature, 434, 871 
Freytag, B., Steffen, M. \& Dorch, B. 2002, AN, 323, 213

Goldman, A., \& Gillis J. R. 1981, JQSRT, 25, 111

González Hernández, J., Bonifacio, P., Ludwig, H.-G., et al. 2008, A\&A, 480, 233

Gratton, R. G., Carretta, E., Eriksson, K., \& Gustafsson, B. 1999, A\&A, 350, 955

Herwig, F. 2004, ApJS, 155, 651

Hill, V., Barbuy, B., Spite, M., et al. 2000, A\&A, 353, 557

Ivarsson, S., Litzén, U., \& Wahlgren, G. M. 2001, Phys. Scr., 64, 455

Johnson, J. A., \& Bolte, M. 2004, ApJ, 605, 462

Jonsell, K., Barklem, P. S., Gustafsson, B., et al. 2006, A\&A, 451, 651

Karakas, A. I., \& Lattanzio, J. C. 2007, PASA, 24, 103

Kurucz, R. 1993, ATLAS9 Stellar Atmosphere Programs and $2 \mathrm{~km} \mathrm{~s}^{-1}$ grid. Kurucz CD-ROM (Cambridge, Mass.: Smithsonian Astrophysical Observatory), 13

Kurucz, R. L. 1996, in ed. K. G. Strassmeier, \& J. L. Linsky (Dordrecht: Kluwer), IAU Symp., 176, 523

Kurucz, R. L. 2005a, MSAIS, 8, 14

Kurucz, R. L. 2005b, kurucz . harvard . edu/

Lawler, J. E., Bonvallet, G., \& Sneden, C. 2001a, ApJ, 556, 452

Lawler, J. E., Wickliffe, M. E., Cowley, C. R., \& Sneden, C. 2001b, ApJS, 137, 341

Lawler, J. E., Den Hartog, E. A., Sneden, C., \& Cowan, J. J. 2006, ApJS, 162, 227

Lawler, J. E., Den Hartog, E. A., Labby, Z. E., et al. 2007, ApJS, 169, 120

Lawler, J. E., Sneden, C., Cowan, J. J., et al. 2008, ApJS, 178, 71

Lawler, J. E., Sneden, C., Cowan, J. J., Ivans, I. I., \& Den Hartog, E. A. 2009, ApJS, 182, 51

Li, R., Chatelain, R., Holt, R. A., et al. 2007, Phys. Scr., 76, 577

Lodders, K. 2003, ApJ, 591, 1220

Lucatello, S., Tsangarides, S., Beers, T. C., et al. 2005, ApJ, 625, 825

Ludwig, H.-G., Caffau, E., Steffen, M., et al. 2009, MmSAI, 80, 711
Lucatello, S., Beers, T. C., Christlieb, N., et al. 2006, ApJ, 652, L37

Marigo, P. 2001, A\&A, 370, 194

McWilliam, A. 1998, AJ, 115, 1640

Mihalas, D. 1978, Stellar atmospheres (San Francisco: W. H. Freeman \& Co.), 2nd edn

Norris, J. E., Ryan, S. G., \& Beers, T. C. 1997, ApJ, 488, 350

Norris, J. E., Christlieb, N., Korn, A. J., et al. 2007, ApJ, 670, 774

Paunzen, E., Kamp, I., Iliev, I. Kh., et al. 1999, A\&A, 345, 597

Reyniers, M., Van Winckel, H., Biémont, E., \& Quinet, P. 2002, A\&A, 395, 35

Ryan, S. G. 1998, A\&A, 331, 1051

Sbordone, L. 2005, MSAIS, 8, 61

Sbordone, L., Bonifacio, P., Castelli, F., \& Kurucz, R. L. 2004, MSAIS, 5, 93

Sivarani, T., Beers, T. C., Bonifacio, P., et al. 2006, A\&A, 459, 125

Sneden, C., Cowan, J. J., Lawler, J. E., et al. 2003, ApJ, 591, 936

Spite, M., \& Spite, F. 1982a, Nature, 297, 483

Spite, F., \& Spite, M. 1982b, A\&A, 115, 357

Spite, M., Cayrel, R., Plez, B., et al., 2005, A\&A, 430, 655

Stancliffe, R. J. 2009, MNRAS, 394, 1051

Steenbock, W., \& Holweger, H. 1984, A\&A, 130, 319

Stehlé, C., \& Hutcheon, R. 1999, A\&AS, 140, 93

Stürenburg, S., \& Holweger, H. 1990, A\&A, 237, 125

Thompson, I. B., Ivans, I. I., Bisterzo, S., et al. 2008, ApJ, 677, 556

Ventura, P., \& Marigo, P. 2009, MNRAS, 399, L54

Ventura, P., D’Antona, F., \& Mazzitelli, I. 2002, A\&A, 393, 215

York, D. G., Adelman, J., Anderson, J. E., et al. 2000, AJ, 120, 1579

Wahlgren, G. M. 2005, MSAIS, 8, 108

Wedemeyer, S., Freytag, B., Steffen, M., Ludwig, H.-G., \& Holweger, H. 2004, A\&A, 414, 1121

Wickliffe, M. E., \& Lawler, J. E. 1997, JOSAB, 14, 737

Wickliffe, M. E., Lawler, J. E., \& Nave, G. 2000, JQRST, 66, 363 